\title{
Capillary effects on wave breaking
}

\author{
Luc Deike ${ }^{1,2} \dagger$, Stephane Popinet ${ }^{2,3}$ and W. Kendall Melville ${ }^{1}$. \\ ${ }^{1}$ Scripps Institution of Oceanography, University of California San Diego, La Jolla, California \\ ${ }^{2}$ National Institute for Water and Atmospheric Research, Wellington, New Zealand \\ ${ }^{3}$ Sorbonne Universités, UPMC Univ Paris 06, CNRS, UMR 7190 Institut Jean Le Rond \\ d'Alembert, F-75005 Paris, France.
}

(Received 29 October 2014)

\begin{abstract}
We investigate the influence of capillary effects on wave breaking through direct numerical simulations of the Navier-Stokes equations for a two-phase air-water flow. A parametric study in terms of the Bond number, $B o$, and the initial wave steepness, $\epsilon$, is performed at a relatively high Reynolds number. The onset of wave breaking as a function of these two parameters is determined and a phase diagram in terms of $(\epsilon, B o)$ is presented that distinguishes between non-breaking gravity waves, parasitic capillaries on a gravity wave, spilling breakers and plunging breakers. At high Bond number, a critical steepness $\epsilon_{c}$ defines the wave stability. At low Bond number, the influence of surface tension is quantified through two boundaries separating, firstly gravity-capillary waves and breakers, and secondly spilling and plunging breakers; both boundaries scaling as $\epsilon \sim(1+B o)^{-1 / 3}$. Finally the wave energy dissipation is estimated for each wave regime and the influence of steepness and surface tension effects on the total wave dissipation is discussed. The breaking parameter $b$ is estimated and is found to be in good agreement with experimental results for breaking waves. Moreover, the enhanced dissipation by parasitic capillaries is consistent with the dissipation due to breaking waves.
\end{abstract}

\section{Introduction}

Understanding small-scale wave structures in the ocean is of fundamental importance in order to quantify wave dissipation and air-sea interaction, including gas and momentum exchange, and to improve parametrization for ocean-atmosphere exchange in weather and climate models. The understanding of dissipation induced by breaking waves at various scales has been the topic of intensive research in recent decades, combining field work in the open ocean (Melville 1996; Melville \& Matusov 2002; Veron et al. 2008; Sutherland \& Melville 2013), laboratory experiments on wave breaking (Melville \& Rapp 1985; Tulin \& Waseda 1999; Rapp \& Melville 1990; Melville et al. 2002; Banner \& Peirson 2007; Drazen et al. 2008), and the dissipation induced by smaller scales involving surface tension, like micro-breaking, spilling breaking (Duncan et al. 1999; Duncan 2001; Liu \& Duncan 2003, 2006) and parasitic capillary waves (Perlin et al. 1993; Jiang et al. 1999; Caulliez et al. 1998; Su 1982; Fedorov et al. 1998; Caulliez 2013). The importance of small scales, $O(1-10) \mathrm{cm}$ in the global ocean wave energy fluxes is still an open question and needs further investigation.

Theoretical approaches to describe these wave phenomena have been based mainly on potential theory, and provide insights into wave stability and the onset of wave breaking (McLean 1982; Peregrine et al. 1993; Longuet-Higgins \& Tanaka 1997; Dias \& Kharif 1999). Geometrical, kinematic and dynamical criteria for the onset of wave breaking

$\dagger$ Email address for correspondence: ldeike@ucsd.edu 
have been the subject of constant debate (e.g. the review of Peregrine et al. (1993); Perlin et al. (2013)). Regarding surface tension effects, boundary layer theory has provided a general description of the appearance of capillary ripples on the front of carrier gravity waves (Longuet-Higgins 1963; Fedorov \& Melville 1998; Melville \& Fedorov 2014) and were first observed numerically by Mui \& Dommermuth (1995). Numerical simulations of gravity-capillary waves are based on potential theory with a boundary layer near the interface (Yang \& Tryggvason 1998; Furhman et al. 2004; Fructus et al. 2005), or on Navier Stokes formulation (Mui \& Dommermuth 1995; Tsai \& Hung 2007, 2010), not taking into account the air flow, or the air being modelled by a pressure forcing (Fedorov \& Melville 1998; Melville \& Fedorov 2014). They provide qualitative and quantitative comparisons with the various wave patterns and phenomenology observed experimentally. The enhancement of dissipation by parasitic capillaries has also been described, both experimentally (Zhang 1995; Fedorov et al. 1998; Caulliez 2013) and numerically (Fedorov \& Melville 1998; Tsai \& Hung 2007, 2010; Melville \& Fedorov 2014). Numerical simulations of the complete two-phase flow remain relatively rare and have focused on the resolution of the wave breaking and post impact dynamics, which are numerically challenging (Chen et al. 1999; Song \& Sirviente 2004; Iafrati 2009, 2011; Iafrati et al. 2013). Most of these numerical studies (Chen et al. 1999; Song \& Sirviente 2004; Iafrati 2009,2011 ) use arbitrary steep Stokes wave to study wave breaking, without considering the processes that leads to breaking, as modulation instability, wave focusing or threedimensional instabilities. The use of steep Stokes waves as a toy model for wave breaking process allows to study in detail the wave breaking properties such as the dissipation, the vorticity generation, the air entrainment or the post-breaking flow. The numerical investigation of capillary effects has been limited to wave breaking at high steepness (Song $\&$ Sirviente 2004). The influence of capillary effects on the wave breaking dynamics is discussed extensively experimentally (e.g. the review of Duncan (2001)) while the significance of surface tension effects can be experimentally studied by adding surfactants that are able to modify the breaking kinematic and dissipation properties (Liu \& Duncan 2003, 2006).

Most recently Melville \& Fedorov (2014) using the theory of Fedorov \& Melville (1998) have shown that the dissipative effects of parasitic capillaries on short, $O(1-10) \mathrm{cm}$, gravity-capillary waves may be enough to balance the wind input and also be consistent with the inertial scaling of the breaking parameter, $b$, presented first for plunging waves by Drazen et al. (2008), and extended by Romero et al. (2012) to encompass the full range of breaking strengths, including spilling breakers (Pizzo \& Melville 2013).

In this paper, we present two-dimensional direct numerical simulations of the NavierStokes equations in the two phases, air and water. The wave dynamics is described as a function of the initial wave steepness and the Bond number (the ratio between gravity and surface tension forces). Various small-scale wave patterns are observed: non-breaking gravity waves, parasitic capillary ripples on gravity waves, spilling breakers and plunging breakers. We investigate the influence of surface tension and the initial steepness on the various wave regimes. All these regimes are obtained by changing the initial steepness of the wave and the Bond number while the Reynolds number, the density ratio and the viscosity ratio are kept constant. A wave-state diagram is presented and the asymptotic critical steepness at high Bond number is compared to theoretical and experimental results. At low Bond number, the influence of surface tension is quantified through two boundaries separating firstly gravity-capillary waves and breakers, and secondly spilling and plunging breakers. Both boundaries are found to scale as $\epsilon \sim(1+B o)^{-1 / 3}$, which can be explained by balancing the vorticity generated by gravity-capillary waves (LonguetHiggins 1992) and the pressure due to surface tension effects. Finally, a description of 
the wave dissipation for the various wave states is presented and capillary effects on wave dissipation are discussed. The dissipation rate during the breaking stages is estimated and the breaking parameter $b$ defined by Duncan (1981) and Phillips (1985) is compared with experimental results (Drazen et al. 2008; Romero et al. 2012; Grare et al. 2013). Very good agreement between the numerical results presented here and the available experimental data is found for plunging and spilling breakers and the dissipation due to parasitic capillaries is found to be comparable with the dissipation by breaking waves.

This paper is organized as follows. First we present the configuration of the numerical experiment in part 2: the flow solver Gerris is briefly introduced, the physical parameters of gravity-capillary waves are described, and the classical potential theory of water waves which is used to define the initial conditions is reviewed. In part 3 we first describe the various wave regimes obtained for different values of the Bond number and the initial wave steepness. Then the parametric study is presented and the wave state is systematically investigated as a function of the initial steepness and Bond number. The wave state diagram is then presented and discussed. Finally in part 4 the energy dissipation for the various wave states is discussed. Comparisons between the wave regime diagram and existing experiments are discussed in part 5 and conclusions are presented in part 6 .

\section{Configuration of the numerical experiment}

\subsection{Gerris flow solver}

The open source flow solver Gerris described in Popinet (2003, 2009) is used is this study. Gerris is based on a quad/octree adaptive spatial discretization, multilevel Poisson solver. The full Navier-Stokes equations in a two-phase flow are solved in 2D, including surface tension. The interface between the high density liquid (water) and the low density gas (air) is reconstructed by a Volume Of Fluid (VOF) method. The multifluid interface is traced by a function $\mathcal{T}(\vec{x}, t)$, defined as the volume fraction of a given fluid in each cell of the computational mesh. The density and viscosity can thus be written as $\rho(\mathcal{T})=$ $\mathcal{T} \rho_{w}+(1-\mathcal{T}) \rho_{a}, \mu(\mathcal{T})=\mathcal{T} \mu_{w}+(1-\mathcal{T}) \mu_{a}$, with $\rho_{w}, \rho_{a}$ and $\mu_{w}, \mu_{a}$ the density and viscosity of the two fluids (water and air), respectively. A detailed description of the numerical methods can be found in Popinet $(2003,2009)$. This solver has been used recently to study complex phenomena involving multiphase flow, like atomization processes (Fuster et al. 2009; Agbaglah et al. 2011). It has been validated extensively, in particular through comparisons with wave-like solutions for capillary waves (Popinet 2009) as well as shearlayer instabilities (Bague et al. (2010); Fuster et al. (2013)). The incompressible, variable density, Navier-Stokes equations with surface tension can be written

$$
\begin{gathered}
\rho\left(\partial_{t} \vec{u}+(\vec{u} \cdot \vec{\nabla}) \vec{u}\right)=-\vec{\nabla} p+\nabla \cdot(2 \mu \vec{D})+\gamma \kappa \delta_{S} \vec{n} \\
\partial_{t} \rho+\nabla \cdot(\rho \vec{u})=0 \\
\nabla \cdot \vec{u}=0
\end{gathered}
$$

with $\vec{u}=(u, v, w)$ the fluid velocity, $\rho \equiv \rho(\vec{x}, t)$ the fluid density, $\mu \equiv \mu(\vec{x}, t)$ the dynamic viscosity and $\vec{D}$ the deformation tensor defined as $D_{i j} \equiv\left(\partial_{i} u_{j}+\partial_{j} u_{i}\right) / 2$. The Dirac delta, $\delta_{S}$, expresses the fact that the surface tension term is concentrated on the interface, where $\gamma$ is the surface tension coefficient, $\kappa$ and $\vec{n}$ the curvature and normal to the interface. Surface tension in Eq. (2.1) is solved explicitely using the method described in Popinet (2009). The computations are performed with a uniform surface tension.

Current problems about computational time in fluid flow modeling are linked to the wide range of spatial scales often encountered. Consequently, adaptive mesh refinement, where the spatial discretization is adjusted to follow the scale and temporal evolution of 
flow structures presents evident benefits. The spatial discretization uses a quad (2D) or octree (3D) scheme. Each cell may be the parent of either four (2D) or eight (3D) children. The root cell is the base of the tree and a leaf cell is a cell without child. The level of a cell is defined by starting from zero for the root cell and by adding one every time a group of four descendant children is added. We will call the maximum grid level $L_{M A X}$. It gives the finest refinement used in the simulation. In surface wave simulations we need to have a high resolution of the interface and the boundary layer where energy will be dissipated. Thus the chosen refinement criteria focus on the flow vorticity and the interface between the liquid and the gas phases. Further away from the interface, the resolution decreases since the quasi-irrotational motion can be appropriately described with a coarser mesh. The parameter $L_{M A X}$ allows comparison of our results to simulations with a fixed grid. For example, our $L_{M A X}=9\left(2^{9}=512\right)$ is equivalent to the $512 \times 512$ grid used in previous studies for direct numerical simulations of breaking waves (Song \& Sirviente (2004); Iafrati (2011)). We have checked that no significant changes are observed when the maximum grid level is increased to $L_{M A X}=10$, thus all results presented here on the wave energy evolution and the interface dynamics have converged regarding the grid resolution.

\subsection{Physical parameters of gravity-capillary waves}

Two-dimensional simulations of surface waves are computed, in a two-phase flow: the liquid on the bottom and the gas on the top. Therefore, within the 2D constraint, we capture naturally all the physical phenomena taking place at the interface without resorting to any simplification of the model. We are solving the complete Navier-Stokes equations, including surface tension and viscosity (see eq. 2.1) to investigate the capillary effects on breaking waves.

A wave is initialized and propagates in the $x$ direction with periodic boundary conditions. The top and bottom walls are free-slip. The wavelength of the initial wave is the length of the box. The study is conducted in terms of dimensionless parameters, with the wavelength and gravity set to unity, $\lambda=1, g=1$. The parameters of the problem with a finite depth are then defined as follows: i) The air-water density ratio $\alpha=\rho_{a} / \rho_{w}=1 / 850$. ii) The air-water dynamic viscosity ratio $\beta=\nu_{a} / \nu_{w}=17.4 \times 10^{-6} / 8.9 \times 10^{-4}$. iii) The Reynolds number in the water $R e=c L / \nu_{w}$, with $L=\lambda$ the length scale, $c$ the linear gravity wave phase speed $c=\sqrt{g / k}$, with $k=2 \pi / \lambda$ the wave number. Consequently $R e=\sqrt{g \lambda^{3}} / \nu$ and we keep the Reynolds number constant in all the simulations presented here, with $R e=40000$. This choice is a compromize between our desire to work at high Reynolds number in order to focus on the effects of the wave amplitude and surface tension rather than that of viscosity, and the grid resolution necessary to capture the viscous processes. iv) The depth ratio $h / \lambda=1 / 2$ with $h$ the depth of the water and $L$ the horizontal box size. The depth effects on the wave evolution are negligible, which was checked with simulations for larger $h$. v) The initial steepness $\epsilon=a k$, with $a$ the initial amplitude of the wave and $k$ the wave number. vi) The Bond number $B o=\Delta \rho g /\left(\gamma k^{2}\right)$, with $\Delta \rho$ the density difference between the two fluids and $\gamma$ the surface tension.

The results are presented in non-dimensional units. The evolution in time is normalized by the period of the principal wave $T=1 / f=2 \pi / \sqrt{g k}$, given by the linear dispersion

relation of the main gravity wave. The two dimensional vorticity field $\Gamma=\frac{\partial v}{\partial x}-\frac{\partial u}{\partial y}$ is normalized by $\Gamma_{0}=\sqrt{g \lambda} / \lambda(\vec{u}=(u, v)$ denotes the velocity field $)$.

We will now describe the initial conditions of the wave simulations. 
2.3. Initialization with a third order Stokes solution.

The simulation is initialized with a modified potential solution for gravity waves, a third order Stokes wave. Note that this solution does not include surface tension effects. Considering a free surface over a liquid of depth $h, x$ the horizontal direction and $y$ the vertical direction, the initial fluid flow is assumed to be irrotational. Hence, we have (Lamb 1932),

$$
\begin{array}{r}
\frac{\partial^{2} \phi}{\partial x^{2}}+\frac{\partial^{2} \phi}{\partial y^{2}}=0,-h<y<\eta \\
\frac{\partial \phi}{\partial y}=0 \text { as } y=-h \\
\frac{\partial \phi}{\partial t}+g \eta+\frac{1}{2}\left(\left(\frac{\partial \phi}{\partial x}\right)^{2}+\left(\frac{\partial \phi}{\partial y}\right)^{2}\right)=\frac{1}{2} c^{2} \\
\frac{\partial \eta}{\partial t}+\frac{\partial \phi}{\partial x} \frac{\partial \eta}{\partial x}+\frac{\partial \phi}{\partial y}=0
\end{array}
$$

where $\phi(x, y, t)$ is the velocity potential, $y=\eta(x, y, t)$ is the free surface, $c$ the phase speed of the unperturbed wave of wavelength $\lambda$, and $g$ the acceleration of gravity. These equations admit, in the frame of reference moving with the wave, a steady solution of the form of a sum over all Fourier components (McLean 1982). Equations (2.2) can be solved numerically (Fenton 1988), and the complete Stokes wave can be used in potential simulations (Furhman et al. 2004; Fructus et al. 2005; Tsai \& Hung 2007). Following previous work on breaking waves (Chen et al. 1999; Song \& Sirviente 2004; Iafrati 2011), we use the third order solution of equations (2.2) that can be solved explicitely through a perturbation approach (Lamb 1932). At the third order in $\varepsilon$, with a fixed bottom $-h$, the interface elevation is given by Lamb (1932):

$$
\begin{aligned}
\eta & =a_{0} \cos (k x)+\varepsilon \frac{a_{0}}{4} \chi\left(3 \chi^{2}-1\right) \cos (2 k x) \\
& +\varepsilon^{2} a_{0}\left(-\frac{3}{8}\left(\chi^{4}-3 \chi^{2}+3\right) \cos (k x)+\frac{3}{64}\left(8 \chi^{6}+\left(\chi^{2}-1\right)^{2}\right) \cos (3 k x)\right)
\end{aligned}
$$

where $\chi=1 / \tanh (k h)$. The velocity potential is given by:

$$
\begin{aligned}
\phi & =\frac{a_{0} g}{\omega} \frac{\cosh (k(y+h))}{\cosh (k h)} \sin (k x) \\
& +\varepsilon \frac{3 a_{0} g}{\omega} \frac{\left(\chi^{2}-1\right)^{2}}{8 \chi} \frac{\cosh (2 k(y+h))}{\cosh (2 k h)} \sin (2 k x) \\
& +\varepsilon^{2} \frac{a_{0} g}{64 \omega}\left(\chi^{2}-1\right)\left(\chi^{2}+3\right)\left(9 \chi^{2}-13\right) \frac{\cosh (3 k(y+h))}{\cosh (3 k h)} \sin (3 k x)
\end{aligned}
$$

where $\omega=\sqrt{g k \tanh (k h)\left(1+\varepsilon^{2}\left(\frac{9}{8}\left(\chi^{2}-1\right)^{2}+\chi^{2}\right)\right)}$ is the dispersion relation at the third-order, and $\varepsilon=a_{0} k$ the steepness of the linear wave and the small parameter of the non-linear expansion. Previous studies have discussed the use of a third order solutions as initial conditions being relevant for the simulation of breaking waves (Chen et al. 1999; Iafrati 2011).

The velocity potential in the liquid is obtained assuming a free surface, i.e the variation in the gas pressure is negligible. In our two-phase simulations the gas phase also has to be initialized. Fixing the initial velocity in the air to zero creates too much shear and the interface becomes unstable due to Kelvin-Helmoltz instabilities. To obtain a smoother, 
more physical initial condition, we follow Yang \& Tryggvason (1998) and add a vortex sheet compatible with the potential solution described above.

A thin vorticity field $\Omega$ at the interface is initialized and the Poisson equation $\nabla^{2} \Psi=\Omega$, with $\Psi$ the stream function, is solved to obtain the initial velocity field in both phases. The initial vortex sheet is of the form $\Omega=\Gamma_{I} \delta_{S}$, with $\delta_{S}$ a Dirac distribution on the interface and $\Gamma_{I}$ the initial vorticity. Following Schwartz (1966), the initial vorticity field, compatible with the velocity potential in the liquid given by eq. (2.4) and a steady state in the gas with no velocity can be expressed as:

$$
\Gamma_{I}=-2 \frac{\partial \Psi}{\partial y} \delta_{S}(\eta(x)-y)=2 \frac{\partial \phi}{\partial x} \delta_{S}(\eta(x)-y) .
$$

The Dirac distribution of the previous equation has to be modeled with a finite boundary layer. Considering the steady Navier-Stokes equations with viscosity and the condition of incompressibility, and assuming the boundary layer thickness $\delta$ to be much smaller than the wavelength $\lambda$, we get (Batchelor (1967)) $\delta \approx \frac{\lambda}{\sqrt{R e}}$. So an asymmetric Gaussian distribution is used to describe the Dirac distribution with a thickness given by the boundary layer $\delta$ : in the water $\delta_{w}=\sqrt{2 \nu_{l} / c k}$ and in the air $\delta_{a}=\sqrt{2 \nu g / c k}$. Thus the boundary layer thickness in the air is $\sqrt{\nu_{w} / \nu a} \approx 4$ times larger than that in the water. Note that this hypothesis is in agreement with Lamb (1932) which shows that for wave oscillations under the influence of viscosity, vorticity remains in a thin layer $\delta=\sqrt{2 \nu / c k}$ and this thickness was previously used to study gravity-capillary waves and develop a boundary layer theory where boundary conditions outside the layer are given by the potential theory (Longuet-Higgins 1992; Fedorov \& Melville 1998). Finally the Dirac distribution is approximated by:

$\delta_{S}(y-\eta) \approx D(y-\eta)=\frac{2}{\sqrt{2 \pi \delta_{a}^{2}}+\sqrt{2 \pi \delta_{w}^{2}}}\left(T \exp -\frac{(y-\eta(x))^{2}}{2 \delta_{w}}+(1-T) \exp -\frac{(y-\eta(x))^{2}}{2 \delta_{a}}\right)$

where $\mathcal{T}$ is equal to 1 in the liquid and 0 in the gas. $D(y-\eta)$ is numerically evaluated with the maximal grid resolution, both in $x$ and $y$ directions, i.e. $d x=d y=\lambda / 2^{9}$.

This initialization allows the simulation of surface waves with various Bond numbers, Reynolds numbers and slopes. Note again that surface tension is not taken into account in the initial conditions and will act once the wave starts to propagate.

\section{Gravity-capillary waves: parametric study as a function of the Bond number and initial steepness}

\subsection{Wave patterns}

We will now describe the waves obtained for different Bond numbers $B o$ and initial steepnesses $\epsilon$, at $R e=40000$. This permits us to investigate the effects of surface tension while the viscosity remains constant.

\subsubsection{Non-breaking gravity wave}

Figure 1 shows two examples of gravity waves with negligible surface tension $(B o=$ 1000) and initial steepness, $\epsilon=0.2$ and $\epsilon=0.25$. The black line corresponds to the interface between the liquid (bottom) and the gas (top). The color scale represents the two dimensional normalized vorticity field $\Gamma^{*}=\Gamma / \Gamma_{0}$. In both cases, the wave propagates with a decreasing amplitude due to viscous damping, with the wave profile becoming steeper during the propagation. The wave becomes more asymmetric when the initial steepness is higher and a stronger vorticity field is also observed both in the air and 
(a) $t / T=1$

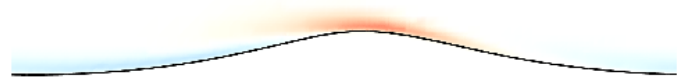

(c) $t / T=1$

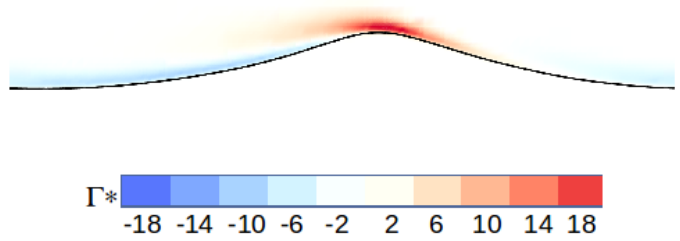

(b) $t / T=1$

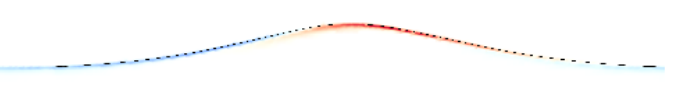

(d) $t / T=1$

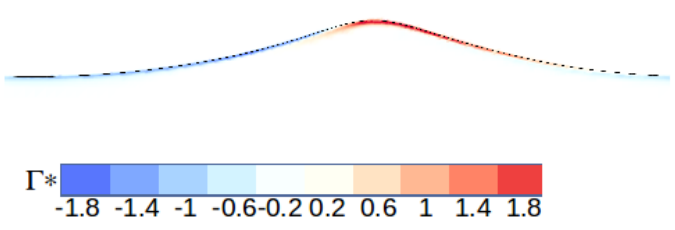

Figure 1: Non-breaking gravity waves propagating to the right and the vorticity field $\Gamma^{*}=\Gamma / \Gamma_{0}$ at $\mathrm{t} / \mathrm{T}=1$, for two different steepness: $0.2(\mathrm{a}, \mathrm{b})$ and $0.25(\mathrm{c}, \mathrm{d})$. The vorticity field is displayed both in the air and water (left) and only in the water (right) with different color-scale. $R e=4 \times 10^{4}, B o=1000$. We can see that the wave is more asymmetric for $\epsilon=0.25$, due to stronger non-linear effects.

water. Both effects are the signatures of non-linearity and can be seen as the beginning of the process that will eventually lead to wave breaking for higher steepnesses. The vorticity field in the water remains located in the thin boundary layer. Moreover, a steeper wave propagates faster due to non-linear effects Lamb (1932). However, for these relatively low initial steepnesses, the wave remains stable (non breaking) and propagates smoothly without dramatic changes of the wave shape. The vorticity remains localized at the interface, within a thin boundary layer and deeper into the liquid the fluid motion remains irrotational. Dissipation processes are consequently localized near the interface as expected for waves of small amplitude Lamb (1932). These waves are obtained for small slopes and high Bond numbers.

\subsubsection{Plunging breaker}

Figure 2 shows a typical plunging wave, obtained for $\epsilon=0.55$ and with almost no surface tension, $B o=1000$. The formation and the evolution of the plunging breaker is similar to the description of plunging breakers observed in laboratory experiments, e.g. Rapp \& Melville (1990): the wave becomes steeper, the interface becomes vertical and a jet forms toward the crest. Then under the influence of gravity the jet splashes down into the surface causing air entrainment and vortical motion beneath the surface. Liquid droplets and gas bubbles are formed when the overturning wave hits the liquid. Plunging breakers generate significant air entrainment when the jet reconnects to the liquid surface. During the breaking process the positive (anti-clockwise) vorticity remains located at the crest (the tip of the jet), and is stronger than the negative vorticity in the trough. We can also observe separation of the vorticity layer in the air behind the crest. There is a dramatic change in vorticity after the jet reentry to the water since vorticity is created during the overturning. The vorticity of largest magnitude is observed during the splash up phenomenon. Vorticity is created by viscous shear torque (Chen et al. 1999) and by generation of vorticity close to high-curvature regions of the interface (Batchelor 1967; Longuet-Higgins 1992). The plunging of the jet, as well as the splashing process entrains a cavity of air, also generating strong vorticity structures both in the air and water (Iafrati 
(a) $t / T=0.4$

(b) $t / T=0.4$
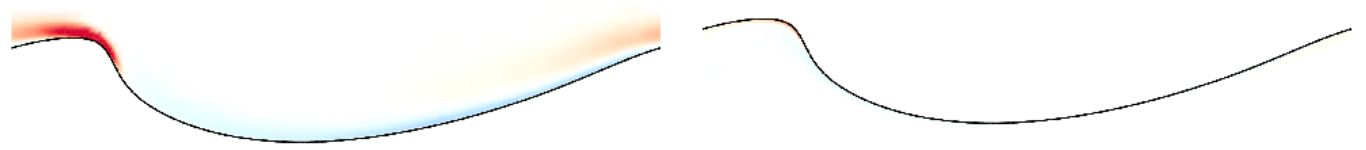

(c) $t / T=0.52$

(d) $t / T=0.52$
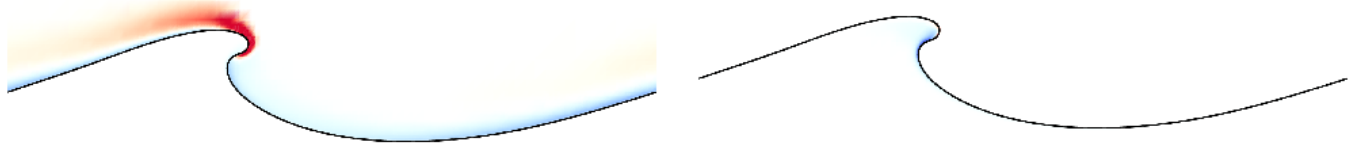

(e) $t / T=0.64$

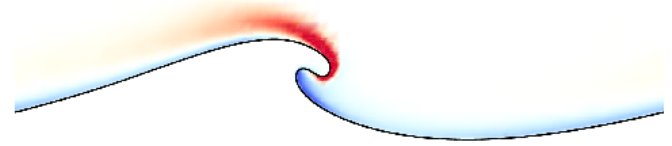

(f) $\mathrm{t} / \mathrm{T}=0.64$

(g) $t / T=0.76$

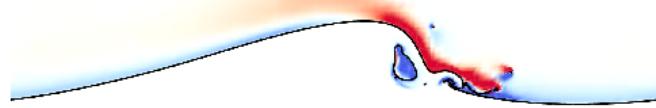

(h) $\mathrm{t} / \mathrm{T}=0.76$
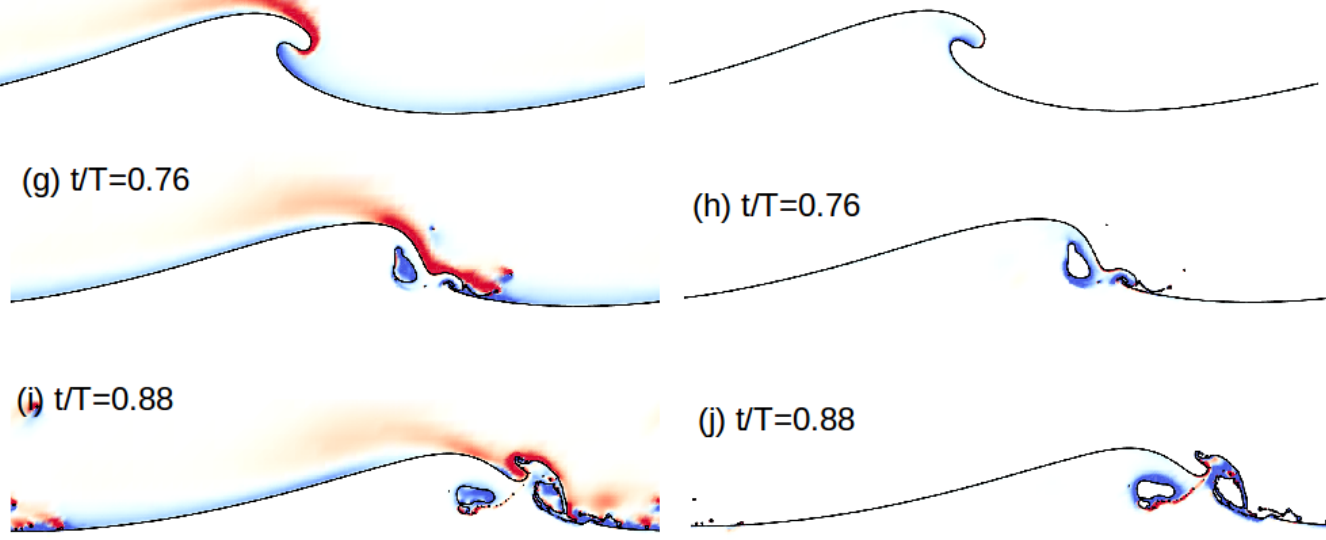

(j) $t / T=0.88$

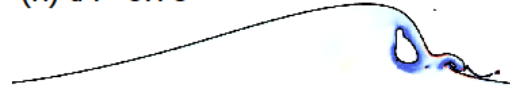

(k) $\mathrm{t} / \mathrm{T}=1.8 \quad$,

(I) $\mathrm{t} / \mathrm{T}=1.8$
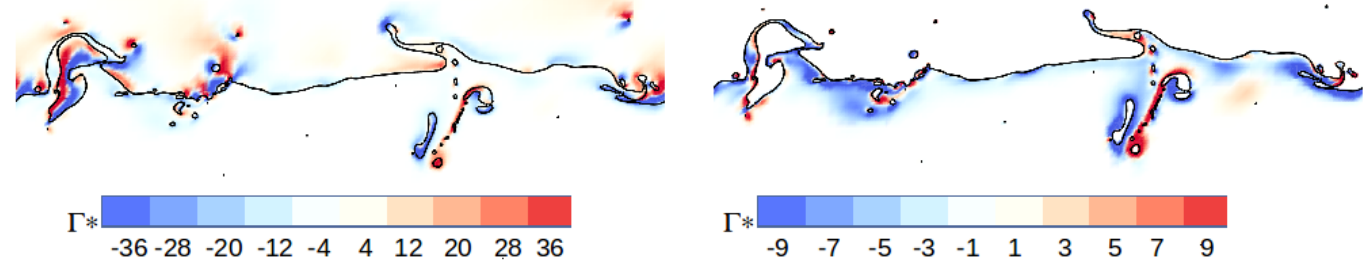

Figure 2: Evolution of a breaking wave and the vorticity field $\Gamma^{*}$, displayed both in the air and water (left) and only in the water (right) with a different color-scale. Initial parameters : $\operatorname{Re}=4 \times 10^{4}, B o=1000, \epsilon=0.55$. The wave is moving from left to right. The wave becomes asymmetric (a-b), leading to a jet formation (c-f), which overturns (e-h), and reconnects $(\mathrm{g}-\mathrm{h})$ to finally splash up $(\mathrm{i}, \mathrm{l})$, where bubbles and spray are generated as well as large vortical structures both in the air and water. 
2009). Vortical structures are also observed following the bubbles in the water and the droplets in the air, related to the change in the free surface topology (Zhang et al. 1993).

Direct numerical simulations of plunging breakers have been previously performed for high initial steepness (Chen et al. 1999; Song \& Sirviente 2004; Iafrati 2009, 2011) and show similar dynamics to that described here. When surface tension is increased (i.e $B o$ decreases), plunging waves are still observed for moderate Bond numbers (not shown) but the jet becomes smoother, leading to a significant reduction of the jet velocity and air entrapment. For these Bond numbers, the surface tension tends to inhibit the wave overturning but this effect is not sufficient to prevent wave breaking. Surface tension effects on plunging waves have been investigated numerically by Song \& Sirviente (2004) for high steepness $(\epsilon>0.5)$ and show similar behavior. Thus, plunging breakers are observed for high initial steepnesses and moderate to high Bond numbers.

\subsubsection{Parasitic capillary waves}

Figure 3 shows the evolution of a wave with strong surface tension effects, $B o=25$, and an initial steepness, $\epsilon=0.3$. The wave behaviour is quite different from the pure gravity wave case described above. Starting with a profile corresponding to a pure gravity wave, parasitic capillaries develop on the forward face of the wave, positive vorticity is located on the main wave crest as well as on the capillary crests while negative vorticity is at the corresponding troughs (both in the air and in the water). Negative vorticity in the trough is higher than positive vorticity on the crest. This mirrors the characteristic asymmetry in curvature of capillary waves (troughs are more curved than crests). We can see the progressive formation of the capillary ripples. The first one appears between $t / T=0.64$ and $t / T=0.76$, vorticity both in the water and in the air being focused on the front of the wave. Then we observe the formation of a train of ripples, with high positive vorticity on the crest and negative vorticity in the through. The capillary train remains stationary in the frame of the main wave and is then simply damped by viscosity. We observe that vorticity remains localized in thin boundary layers in both the air and the water.

These gravity-capillary waves are nonlinear surface waves for which both gravity and surface tension are important, where two typical length scales can be defined, the longer scale corresponds to the main wavelength $(\lambda)$, and the shorter scale to the capillary ripples we observe in the front of the wave $\left(\lambda_{c}=2 \pi \sqrt{\gamma /(g \Delta \rho)}\right)$. Parasitic capillary waves on steep gravity waves have been described theoretically (Longuet-Higgins 1963; Fedorov \& Melville 1998): assuming an underlying pure Stokes wave, high curvature and surface tension effects are localized at the crest of the gravity wave and act as a forcing term. Parasitic capillaries appear initially as a linear response to the local forcing on the Stokes wave and finally are damped by viscosity. In this description, vorticity remains located in a thin boundary layer and parasitic capillaries are responsible for significant dissipation enhancement (Mui \& Dommermuth 1995; Fedorov \& Melville 1998; Melville \& Fedorov 2014).

These waves are observed for initial steepnesses below a breaking threshold and for small Bond number $(B o \lesssim 70)$. Note also that the steepness must be high enough to observe the formation of parasitic capillaries and that the intensity of the vorticity field under the capillaries is related to the wave steepness (Longuet-Higgins 1992). The present behavior is in agreement with the theoretical description given by Longuet-Higgins (1963) and Fedorov \& Melville (1998). 
(a) $t / T=0.64$

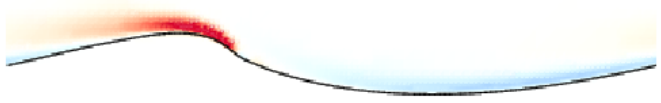

(c) $t / T=0.76$

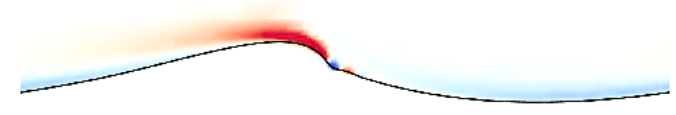

(e) $t / T=0.88$

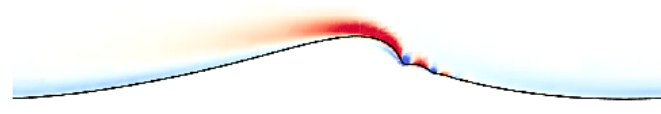

(g) $t / T=1$

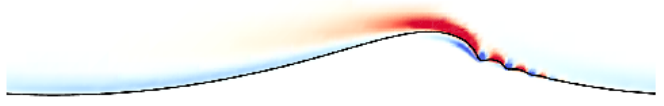

(i) $\mathrm{t} / \mathrm{T}=1.68$

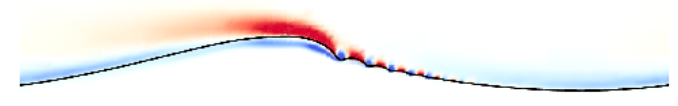

(k) $\mathrm{t} / \mathrm{T}=2.48$
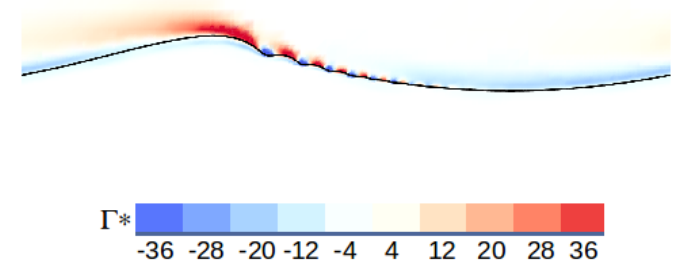

(b) $t / T=0.64$

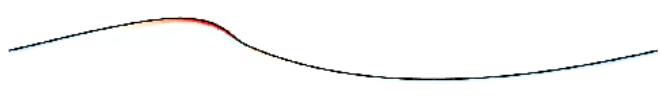

(d) $t / T=0.76$

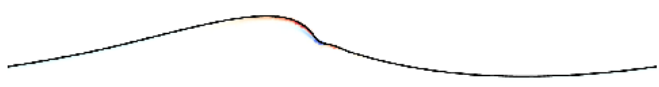

(f) $t / T=0.88$

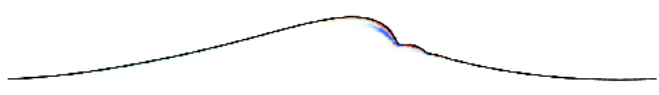

(h) $t / T=1$

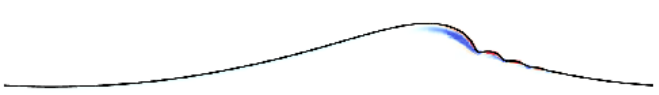

(i) $\mathrm{t} / \mathrm{T}=1.68$

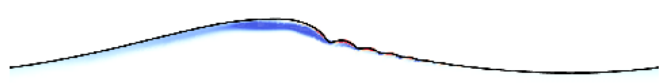

(I) $\mathrm{t} / \mathrm{T}=2.48$
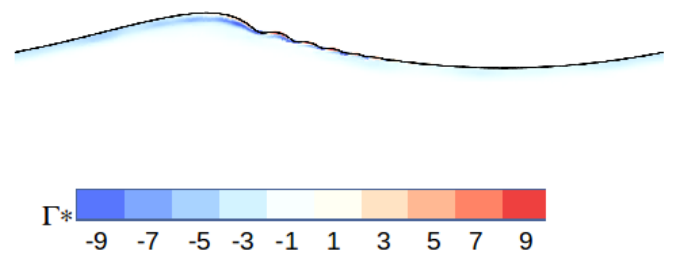

Figure 3: Parasitic capillary waves on a gravity wave and the vorticity field $\Gamma^{*}$, displayed both in the air and water (left) and only in the water (right) with a different color-scale. Initial parameters : $\operatorname{Re}=4 \times 10^{4}, B o=25, \epsilon=0.3$. Parasitic capillaries are generated on the front of the carrier gravity wave when the wave becomes steep enough (a-d). A train of parasitic capillaries is then formed $(\mathrm{c}-\mathrm{h})$ and propagates with the main wave. The amplitude of the carrier decays through viscous effects (i-l). Positive vorticity is located in the crest of the capillaries and negative voricity in the through. 


\subsubsection{Spilling breakers}

Spilling breakers are usually defined as breaking waves without an overturning entrainment of air. The overturning entrainment is avoided either because the slope of the wave is too low, resulting in a gentle spilling wave (Rapp \& Melville 1990), or due to stabilization by surface tension effects (Duncan et al. 1999; Duncan 2001). These waves have been observed and described in laboratory experiments (Rapp \& Melville 1990; Lin \& Rockwell 1995; Duncan et al. 1999; Qiao \& Duncan 2001; Liu \& Duncan 2003, 2006) and numerically by Iafrati \& Campana (2005). This regime is also obtained in our simulations as illustrated in figure 4, for a relative high initial steepness and low Bond number $(B o=25, \epsilon=0.45)$. The spilling breaker dynamics can be described as follows: due to surface tension effects, the free surface does not overturn and the beginning of the breaking process is marked by the formation of a bulge in the profile at the crest on the forward face of the wave (figure $4 \mathrm{a}-\mathrm{d}$ ). The leading edge is sometimes called the "toe of the wave", and as the breaking process continues, the bulge becomes more pronounced while the toe remains in nearly a fixed position relative to the crest (figure $4 \mathrm{c}-\mathrm{f}$ ). Concurrent with the growth of the bulge, capillary waves appear and grow in amplitude upstream of the toe (figure $4 \mathrm{e}-\mathrm{j}$ ). After a short time, the toe begins a rapid motion down the wave face and during this motion a train of large amplitude well organized ripples is formed between the toe and the crest. These ripples grow rapidly and then break down into a random pattern indicating that the underlying flow has become turbulent (figure $4 \mathrm{k}-\mathrm{l})$. Both in the air and water, the vorticity field is positive on the crest and negative in the through. The negative vorticity field in the water appears much stronger, and eventually, is separated due to the turbulent breakdown of the initial boundary layer. Vortex dipoles are therefore generated in the water.

Another example of a spilling breaker, for higher Bond number, is shown in figure 5, $(B o=70, \epsilon=0.45)$. The previous description of a spilling breaker is still valid but the wave is closer to overturning and thus more mixing and aeration is observed. In this case more bubbles are created and the vorticity field in the fluid underneath is more turbulent, and interface reconnection is also observed. Several vortex dipoles are also generated in the water and start to move deeper. Thus it appears that for this intermediate steepness, a small change in the surface tension can cause qualitative changes in the wave dynamics. These types of wave structure changes have been explored experimentally by adding surfacants to water (Liu \& Duncan 2003, 2006). Air entrainment by breaking waves has been investigated experimentally (Lamarre \& Melville 1991, 1994; Loewen et al. 1996; Blenkinsopp \& Chaplin 2007; Rojas \& Loewen 2010) but carefull comparisons would require the extension of the present simulation to three-dimensions.

\subsection{Wave regime diagram}

The different wave regimes observed and described previously are summarized in the state diagram in figure 7 . We first present the criteria chosen to distinguish each of them and then discuss the diagram. The different wave regimes are i) non-breaking gravity waves (phase N-B), ii) parasitic capillary waves on gravity wave (phase P-C-W), iii) plunging breakers (phase P-B), iv) spilling breakers (phase S-B).

Using the criteria defined below, numerical simulations can be used to automatically estimate the critical values of the steepness and Bond numbers for which transitions occur. To do this efficiently, we used an automatic iteration in the $(\epsilon, B o)$ phase space. Simulations are repeated on either side of the transition boundary until convergence. The symbols in Figure 7 are the results of this iterative process. Each symbol on the diagram thus represents the result of typically 5 to 10 simulations. This systematic phase-space exploration was greatly facilitated by the overall speed of the adaptive grid method. 
(a) $t / T=0.4$

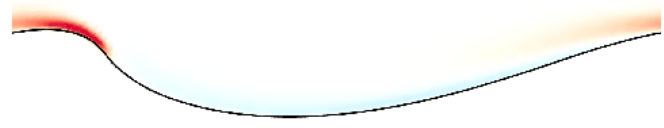

(c) $t / T=0.52$

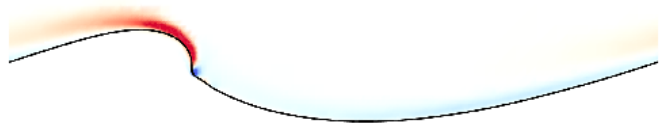

(e) $t / T=0.76$

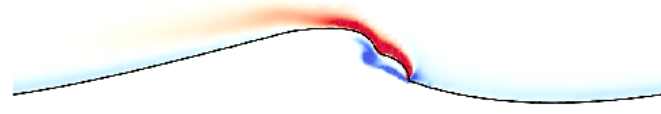

(g) $t / T=0.88$

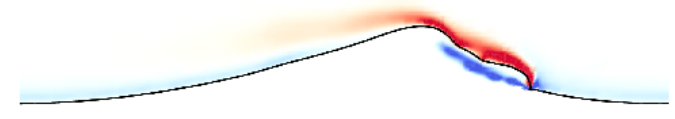

(i) $t / T=1$

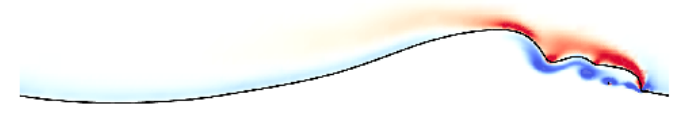

(k) $t / T=1.44$
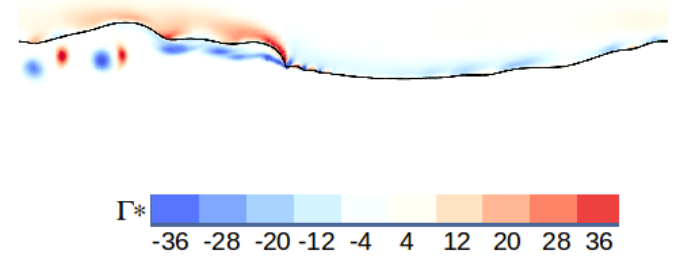

(b) $t / T=0.4$

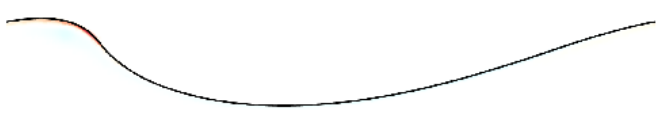

(d) $t / T=0.52$

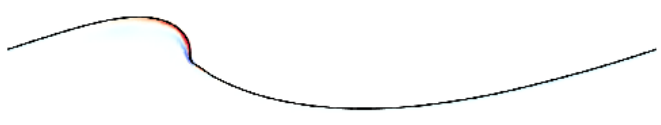

(f) $\mathrm{t} T \mathrm{~T}=0.76$

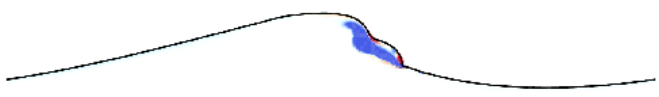

(h) $t / T=0.88$

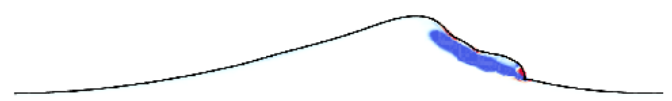

(i) $t / T=1$

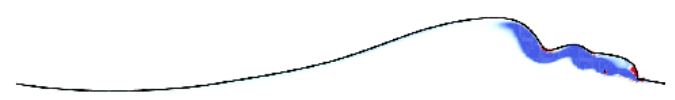

(I) $t / T=1.44$
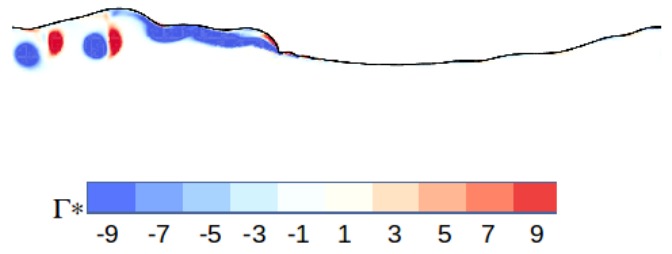

Figure 4: Evolution of a spilling breaker and its vorticity field $\Gamma^{*}$ at different times, displayed both in the air and water (left) and only in the water (right) with different color-scale. Initial conditions $R e=4 \times 10^{4}, B o=25, \epsilon=0.45$. The wave becomes steeper and begins to break (a-d) but the overturning motion is avoided due to capillary effects and capillaries propagate on the front of the toe $(\mathrm{c}-\mathrm{i})$. A separation of the boundary layers in water is observed, without air entrainment but with the formation of vortex dipoles in the water after breaking. 
(a) $t / T=0.52$

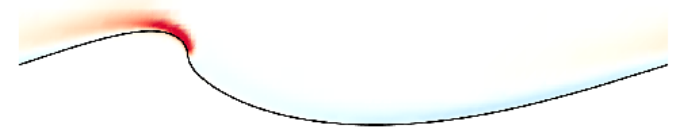

(c) $t / T=0.64$

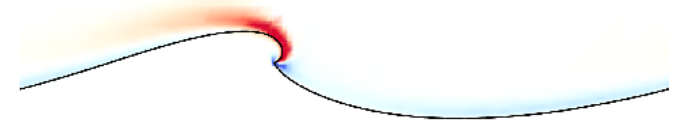

(e) $t / T=0.8$

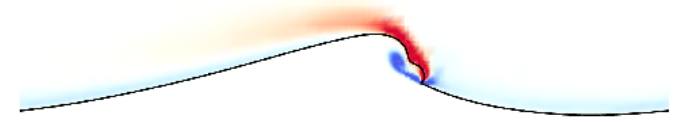

(g) $t / T=1$

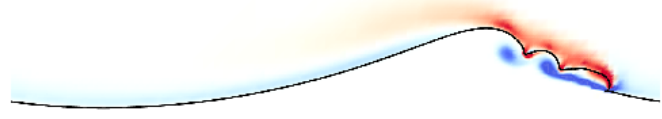

(i) $\mathrm{t} / \mathrm{T}=1.32$

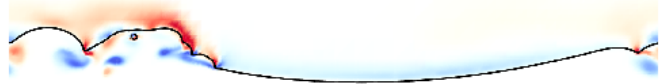

(k) $t / T=2.28$

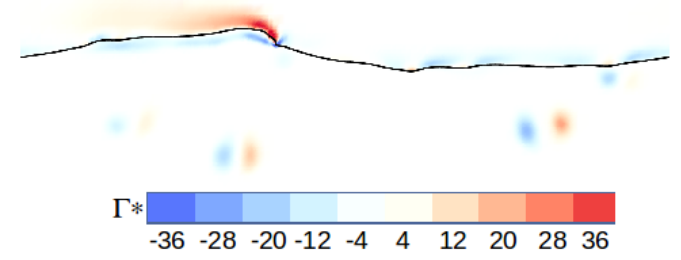

(b) $t / T=0.52$

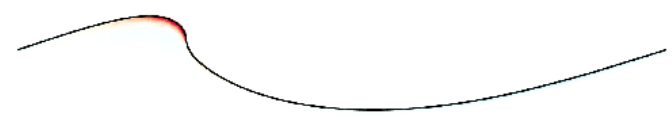

(d) $t / T=0.64$

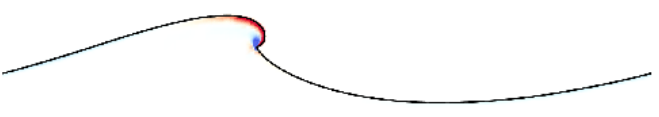

(f) $t / T=0.8$

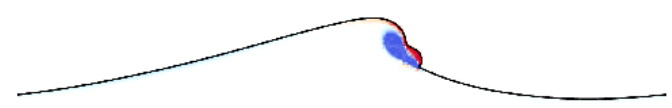

(h) $t / T=1$

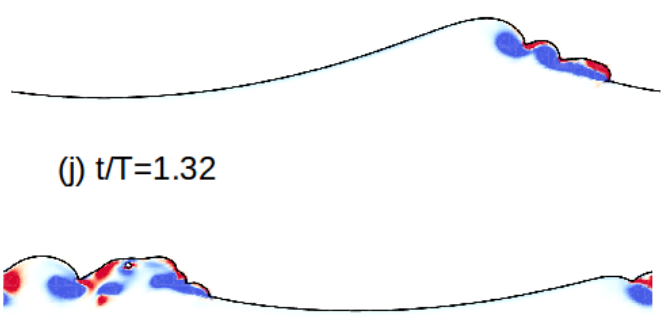

(I) $t / T=2.28$

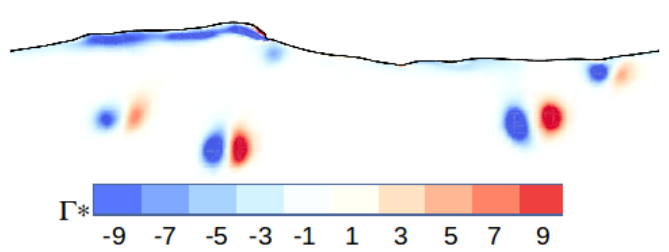

Figure 5: Evolution of a spilling-overturning breaker and its vorticity field $\Gamma^{*}$ at different times, displayed both in the air and water (left) and only in the water (right) with a different color-scale. Initial conditions $R e=4 \times 10^{4}, B o=70, \epsilon=0.45$. The wave begins to break (a-d) similar to figure 4 but with the interface almost overturning (c-d) and formation of bubbles after the breaking stage (i-j). The turbulent breakdown appears stronger and several vortices are generated in the water $(k-1)$. 


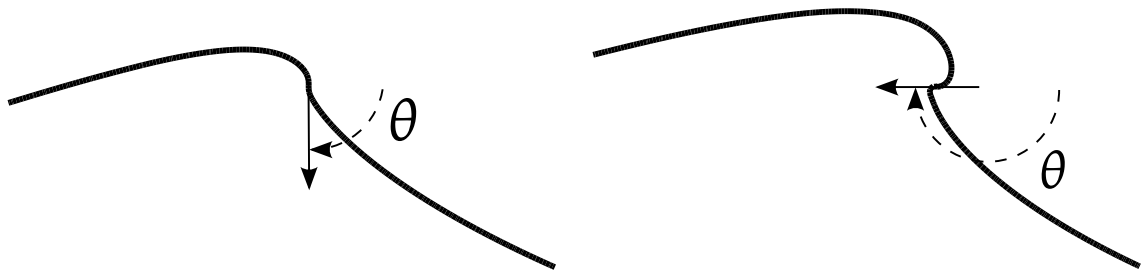

Figure 6: Topological wave state criteria. Left: breaking criterion with the interface presenting a vertical segment (-90 degres angle). Right: overturning wave with the interface presenting both vertical and horizontal segments (-180 degrees angle).

\subsubsection{Wave regime criteria}

Breaking criteria. Vertical interface. Wave breaking criteria have been the subject of strong debates over the years and we choose to characterize the onset of wave breaking by the appearance of a local vertical interface (Peregrine et al. 1993; Perlin et al. 2013). A wave is classified as breaking when it exhibits a vertical interface, as shown on the sketch figure 6 (left). The boundary between non-breaking and breaking waves using this criterion is indicated by ( $\mathbf{\square}$ ) on figure 7 and will be called the breaking boundary in the following. The breaking boundary exhibits two transitions: at high Bond number $(B o \gg 100)$, a critical steepness $\epsilon_{c}=0.32$ determines if the two dimensional wave will break, while at low Bond number, the breaking boundary depends on the Bond number. The wave breaking boundary can be described at high steepness and low Bond number by the following relation: $\epsilon_{B}=K_{B}(1+B o)^{-1 / 3}$, for which we present a physical interpretation in $\S 3.3$ and $K_{B}=1.12$ is a dimensionaless constant fitted on the data. The constant $K_{1}$ can be interpreted as a critical Bond number $B o_{B}=K_{B}^{3}=1.4$ below which no breaking wave can exist.

Overturing wave. Horizontal interface. Plunging and spilling waves (figures 2 and 4) display different interface profiles at and after breaking. Spilling and plunging breakers both exhibit a vertical interface but a plunging wave also exhibits a horizontal interface, as shown in figure 6, and described by Duncan (2001). We choose the presence of an horizontal interface to distinguish plunging and spilling breakers. The plunging breaker in figure 2 satisfies both the breaking (vertical interface) and the overturing wave (horizontal interface) criteria, since a horizontal interface is seen once the jet is formed. On the other hand, the spilling breaker in figure 4 only satisfies the breaking (vertical interface) criterion, since the jet formation is prevented by surface tension effects.

The boundary between spilling and plunging breakers is shown in figure 7 by $\boldsymbol{\nabla}$. At high Bond numbers, the same critical steepness $\epsilon_{c}$ as before is obtained and there is no difference between the breaking boundary and the overturning boundary. At low Bond number and high steepness, the overturning boundary can be described by a similar function as the breaking boundary, $\epsilon_{S P}=K_{S P}(1+B o)^{-1 / 3}$, having the same power law but with a different constant, $K_{S P}=1.75$. Again, the constant $K_{S P}$ can be interpreted as a critical Bond number $B o_{S P}=K_{S P}^{3}=4.8$ below which no plunging breaker can exist.

\subsubsection{Existence of a critical steepness at high Bond number $\epsilon_{c}$}

At high Bond numbers $(B o \gg 100)$ the breaking boundary corresponds to the existence of a critical steepness, here $\epsilon_{c}=0.32$. For $\epsilon<\epsilon_{c}$ the wave does not break (phase $\mathrm{S}$ in figure 7), while for $\epsilon>\epsilon_{c}$ the wave breaks. This critical steepness defines the breaking of a gravity wave and we remark that the breaking and the overturning boundary exhibit the 


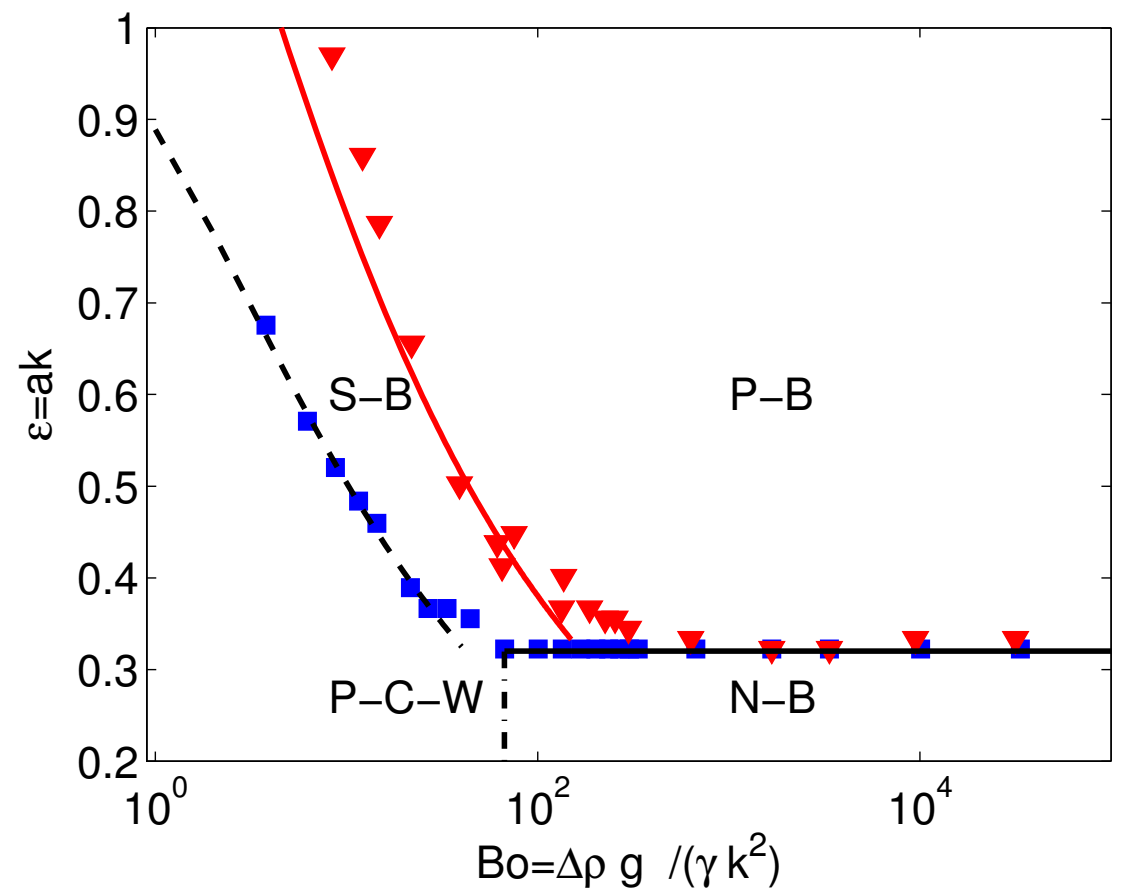

Figure 7: Wave regime diagram $(B o, \epsilon)$. Symbols indicate the boundaries between the wave regimes obtained numerically (see text). $\mathbf{\square}$ : wave breaking boundary, presence of a vertical interface. $\mathbf{\nabla}$ : spilling-plunging boundary, presence of an horizontal interface. Zone (P-B): plunging breakers (as in figure 2); (S-B): spilling breakers (as in figure 4); (P-C-W): parasitic-capillary waves (as in figure 3); (N-B): non-breaking gravity waves (as in figure 1). Horizontal solid line (black) indicates the critical steepness $\epsilon_{c}=0.32$. Black dashed line indicates the boundary between breaking and non-breaking waves: $\epsilon_{B}=K_{B}(1+B o)^{-1 / 3}$, with $K_{B}=1.12$ an adjusted parameter. Solid line (red, light gray) indicates the overturning boundary (between spilling and plunging breakers): $\epsilon_{S P}=$ $K_{S P}(1+B o)^{-1 / 3}$, with $K_{S P}=1.75$ an adjusted parameter. Dot-dashed line: $B o_{c}=67$, the observed critical Bond number for the appearance of parasitic capillaries.

same critical steepness at high Bond number. Thus spilling breakers regime at high Bond number (low surface tension) can be seen as a transition regime between non-breaking and breaking waves. Note that the obtention of a critical steepness for wave breaking is a meaningfull physical result, but the particular value of the critical steepness in idealized numerical simulations strongly depends on the initial conditions.

The finding of an accurate critical steepness for wave breaking remain an open challenge theoretically and has been the scope of many experimental studies (e.g. Perlin et al. (2013)). When breaking is triggered by modulational instabilities, plunging waves can be observed for initial slopes $\epsilon \approx 0.11$ (Melville 1982; Tulin \& Waseda 1999; Song \& Banner 2002; Banner \& Peirson 2007; Iafrati et al. 2013). In focusing experiments a critical slope of the same order is described when considering the dissipation by breaking, and $\epsilon \approx 0.08$ (Drazen et al. 2008; Romero et al. 2012). Note also, that when only considering the shape of the interface as a breaking criterion, higher values of the slope can be considered and $\epsilon \approx 0.25$ (Rapp \& Melville 1990). In these focusing cases, the slope is often evaluated by 
the sum of all linear components of the initial wave packet. Thus the particular value of $\epsilon_{c}$ depends on the initial conditions, on the physical processes that lead to breaking and on the breaking criterion. Note also that in focusing experiments, a dependency in the bandwidth of the wave packet is evident (Rapp \& Melville 1990; Drazen et al. 2008; Banner \& Peirson 2007).

\subsubsection{Parasitic capillaries and spilling breakers at low Bond number, critical steepness breaking curve $\epsilon_{B}(B o)$}

Let us now discuss the breaking wave regimes. At high Bond number and high steepness plunging waves are observed above the critical steepness. Plunging waves are also observed at higher surface tension with a smoother jet. Increasing the surface tension eventually leads to the inhibition of the jet formation and while spilling breakers are observed at high steepness (as in figure 4), parasitic capillaries are observed at relatively low steepness as in figure 3. While spilling breakers satisfy the breaking criterion, parasitic-capillaries are not considered breaking waves.

At high steepness and low Bond number, the breaking boundary is described by a steepness depending on the Bond number, $\epsilon_{B}=K_{B}(1+B o)^{-1 / 3}$, the corresponding function being shown on the state diagram of figure 7 .

Thus, the breaking wave boundary permits us to define the parameter space where parasitic capillaries are present. For $B o>B o_{c}=67$, a critical steepness alone defines the transition from non-breaking to breaking waves. For $B o<B o_{c}$, surface tension stabilizes the wave and a higher initial steepness is needed to observe wave breaking, the breaking to non-breaking wave boundary is described by the $\epsilon_{B}(B o)$ curve. This critical Bond number $B o_{c}$ describes the appearance of parasitic capillary waves: for $B o<B o_{c}$ and $\epsilon<\epsilon_{B}$, we observe the appearance of parasitic capillary waves (as in figure 3), where capillaries can be treated as a perturbation of the main gravity wave (LonguetHiggins 1963; Fedorov \& Melville 1998). Note that according to the Bond number, the corresponding wavelength in the air-water case is $\lambda_{c}=2 \pi \sqrt{\gamma B o_{c} /(\rho g)} \approx 14 \mathrm{~cm}$, which is consistent with the experimental scale where parasitic capillaries are observed for $\lambda \sim O(10 \mathrm{~cm})$ or less (Longuet-Higgins 1963; Fedorov \& Melville 1998).

\subsubsection{Spilling and plunging breakers, critical steepness curve at low Bond number $\epsilon_{S P}(B o)$}

As already noted, at high Bond number the spilling-plunging boundary is described by $\epsilon_{c}$. At low Bond numbers the spilling-plunging boundary is described by the curve $\epsilon_{B}=K_{S P}(1+B o)^{-1 / 3}$. Above the stability boundary and below the plunging boundary, i.e $\epsilon_{B}<\epsilon<\epsilon_{S P}$, spilling breakers (as in figure 4) are observed (zone B-S). The wave still breaks but no overturning is observed. This dynamics is similar to gentle (weak) spilling breakers, observed experimentally (Rapp \& Melville 1990; Duncan 2001).

The fact that for Bond number around 100, the boundary between plunging breakers and spilling breakers is a smooth transition corresponds to the existence of spilling breakers as shown in figure 5, where significant bubble formation and air entrainment appear and thus horizontal interfaces may exist. These highly aerated spilling breakers satisfy both the breaking and the overturning criteria, but without jet formation. Note finally that at high steepness $(\epsilon>0.6)$ and high surface tension, we observe highly non-linear waves similar to pure non-linear capillary waves, with interface reconnection and air entrapment (not shown). These features appear comparable to limiting Crapper waves (Crapper 1957). 


\subsection{A scaling for capillary effects on wave breaking}

We have shown that the separation between breaking and non-breaking waves when surface tension effects are important scales as $\epsilon_{B} \sim(1+B o)^{-1 / 3}$. It is very interesting to notice that this scaling is also valid for separating the spilling and plunging breakers in the $(\epsilon, B o)$ space. We discuss here a possible explanation for this boundary, using the balance between the generation of vorticity by gravity-capillary waves, given by LonguetHiggins (1992) and the Laplace pressure due to the capillary effects $p=\gamma \kappa$, with $\kappa$ the wave curvature.

We want to connect the vorticity generated by the wave to the pressure. Following Douady et al. (1991) and taking the divergence of the Navier Stokes equations in the water (eq. 2.1):

$$
\Gamma^{2}-D_{i j}^{2}=\frac{\nabla^{2} p}{\rho_{w}}
$$

with $\Gamma$ the vorticity and $D_{i j}$ the deformation tensor, linked to the dissipation. Thus the vorticity concentrations will act as source of pressure. At high Reynolds number we have

$D_{i j}^{2} \ll \Gamma^{2}$, which was checked to be valid in our simulations. Thus, one gets the following relation between vorticity and pressure:

$$
\Gamma^{2} \sim \frac{\nabla^{2} p}{\rho_{w}}
$$

We consider the pressure due to to capillary effects,

$$
\nabla^{2} p \sim \gamma k^{4} a
$$

with $a$ the wave amplitude. The vorticity is given by Longuet-Higgins (1992) for a nonlinear gravity-capillary wave:

$$
\Gamma=-2(a k)^{2} \omega
$$

with $\omega=\sqrt{g k+\gamma / \rho k^{3}}$ the dispersion relation. Thus we obtain

$$
(a k)^{4} g k\left(1+\frac{\gamma k^{2}}{g \rho_{w}}\right) \sim \frac{\gamma k^{4} a}{\rho_{w}},
$$

and since the Bond number is given by $B o=\rho_{w} g /\left(\gamma k^{2}\right)$, we finally obtain the following scaling:

$$
(a k) \sim(1+B o)^{-1 / 3}
$$

which corresponds to the boundary between breaking and non-breaking waves when surface tension effects are strong. Thus this simple scaling argument appears to describe the transition from a non-breaking gravity-capillary wave to breakers with strong surface tension and the same scaling holds for describing the transition from spilling breakers to plunging breakers.

\section{Energy dissipation}

4.1. Evolution of the various energy components

We will now discuss the capillary effects on wave dissipation for the various wave patterns described previously. We calculate the various energy components of the propagating wave, the kinetic energy:

$$
E_{k}=\frac{1}{2} \int \rho \vec{u}^{2} d x d y
$$


the gravitational potential energy:

$$
E_{g}=\int \rho g y d x d y+1 / 8,
$$

the surface tension potential energy:

$$
E_{s}=\frac{\gamma}{\rho_{w} g \lambda^{2}}(\mathcal{L}-1),
$$

where $\mathcal{L}$ is the arclength of the interface, and the total energy:

$$
E=E_{k}+E_{g}+E_{s} .
$$

Figure 8 shows the different wave energy components for various parameters $\epsilon$ and $B o$. In all cases, the total wave energy decreases in time due to dissipative processes.

First, we focus on the low surface tension case $(B o=1000)$. When the steepness is below the breaking threshold $(\epsilon<0.32), E$ decreases exponentially at a constant decay rate, as shown by figure $8 \mathrm{a}$, and is close to the theoretical linear viscous decay rate $E / E_{0}=\exp \left(-4 \nu k^{2} t\right)$. The kinetic and potential energies oscillate at half the wave frequency but with opposite phase, showing exchanges between the kinetic and potential energy components, and decay at similar rates. The surface tension potential energy remains negligible throughout the propagation. This case corresponds to a non-breaking gravity wave. For a higher steepness, when wave breaking occurs $(\epsilon=0.45$, figure $8 \mathrm{~b})$, an abrupt decrease of the total energy is observed during the breaking event (starting at $t / T \approx 1$ ), and around $70 \%$ of the wave energy is dissipated in a few wave periods. During the breaking, the gravitational potential energy is almost completely dissipated, and only kinetic energy remains after a few wave periods. Surface tension potential energy remains small during the whole process, even if an increase is observed during the breaking stage, due to high surface curvature, and the generation of droplets and bubbles. The peak value of surface tension energy represents $\approx 8 \%$ of the total dissipated energy of wave, in agreement with results for similar wave parameters from Iafrati (2011). The abrupt decrease of energy during the breaking process can be fitted by an exponential curve between $t / T=1$ and $t / T=2: E=E(t / T=1) \exp (-\zeta t)$, where $\zeta$ is the decay rate during the breaking process and $E(t / T=1)$ is the energy at $t / T=1$. This case corresponds to a plunging breaker.

Chen et al. (1999) studied a plunging breaker at high Bond number and found a decay rate $E \sim e^{-2 \gamma_{2}\left(t-t_{1}\right)}$, where $t_{1}$ is a breaking time and $\gamma_{2}=0.16$, which using our notations gives $\zeta_{\text {Chen }}=2 \gamma_{2}=0.32$. The value of $\zeta$ in our simulation for $B o=1000$ and $\epsilon=0.45$ is $\zeta=0.18$, and for $\epsilon=0.55, \zeta=0.22$, so our values of decay rate are slightly smaller than the one from Chen et al. (1999) for a plunging breaker. This is coherent with the fact that Chen et al. (1999) reports a loss of $80 \%$ of the initial wave energy for a plunging breaker after four wave period while we observe a loss of $\approx 65 \%$. For a similar plunging wave, Iafrati (2011) reports a loss of around $60 \%$ of the energy after four wave period. Note also that for $B o=1000$, the energy after breaking for $\epsilon=0.35$ and $\epsilon=0.45$ is close to the initial energy of the steepest non breaking wave $(\epsilon=0.31)$, in qualitative agreement with the result discussed by Iafrati (2011) that the energy of the residual wave after a spilling breaking process at high Bond number is close to the one of the steepest non-breaking wave.

We now consider the case where surface tension effects become important $(B o<100)$. Figure $8 \mathrm{c}$ shows the evolution of the energy components during wave propagation at relatively low steepness, $(\epsilon=0.3)$ for $B o=50$. During the first wave period, the total energy decays exponentially with the classical decay rate, while at $t / T \approx 1$, the decay 


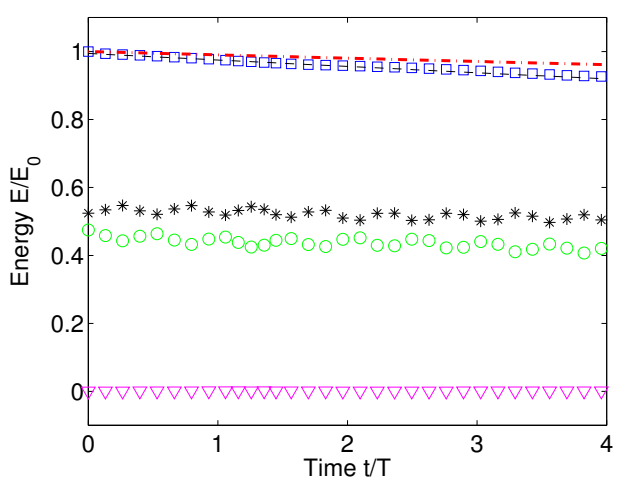

(a) $B o=1000$ and $\epsilon=0.3$

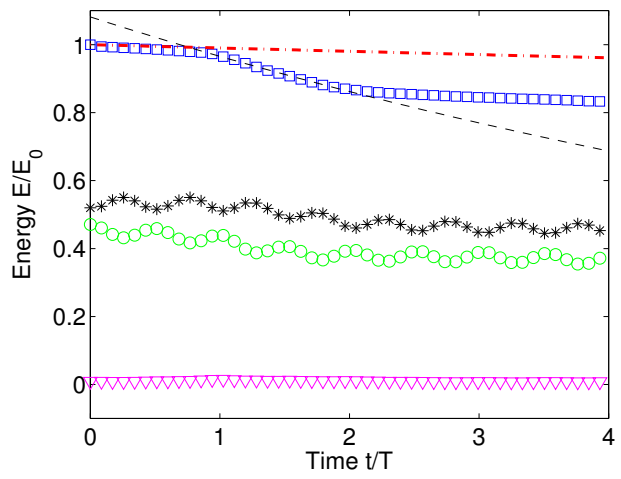

(c) $B o=50$ and $\epsilon=0.3$

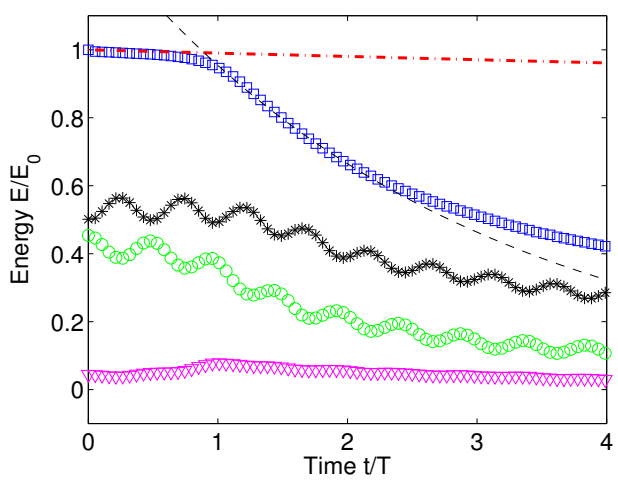

(e) $B o=10$ and $\epsilon=0.3$

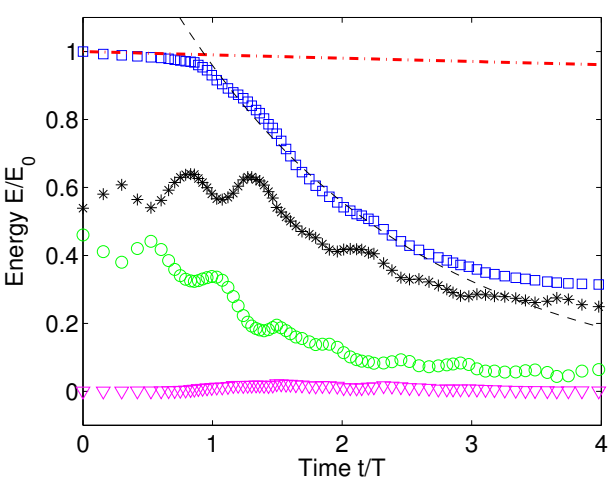

(b) $B o=1000$ and $\epsilon=0.45$

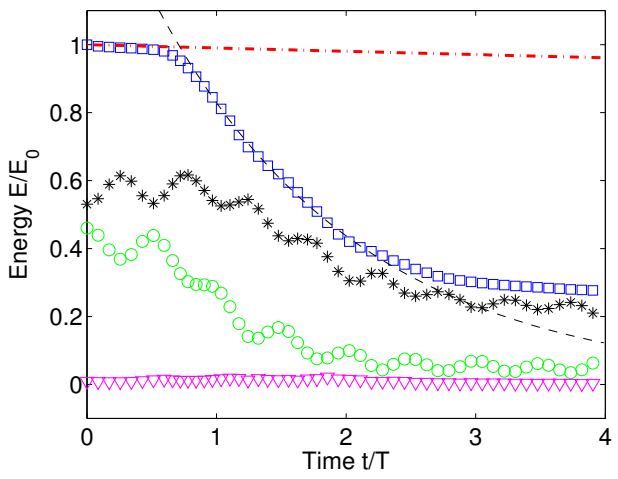

(d) $B o=50$ and $\epsilon=0.45$

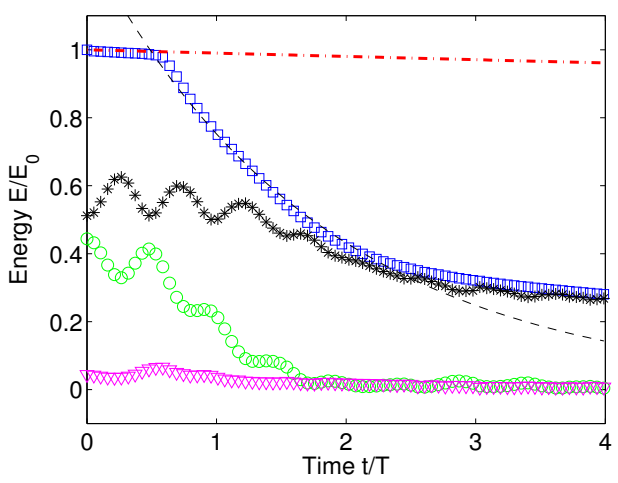

(f) $B o=10$ and $\epsilon=0.45$

Figure 8: Normalized wave energy components as functions of time $t / T$ : total energy $E / E_{0}(\square)$, kinetic energy $E_{k} / E_{0}(*)$, gravitational potential energy $E_{g} / E_{0}(\circ)$ and surface tension potential energy $E_{s} / E_{0}(\nabla)$, for various $B o$ and initial $a k$. $E_{0}$ is the initial total energy. Red dot-dashed line is the theoretical linear viscous dissipation $E / E_{0}=$ $\exp \left(-4 \nu k^{2} t\right)$. Black dashed line is an exponential fit $E=E(t / T=1) \exp (-\zeta t)$ to the energy decay in the time interval $t / T \in[1: 2]$. 


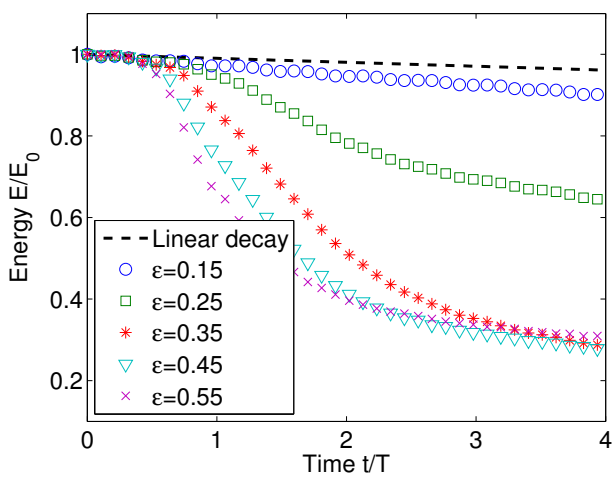

(a) $B o=10$

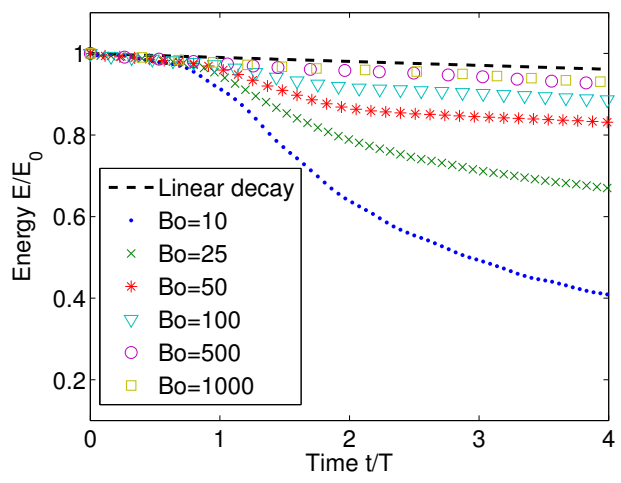

(c) $\epsilon=0.3$

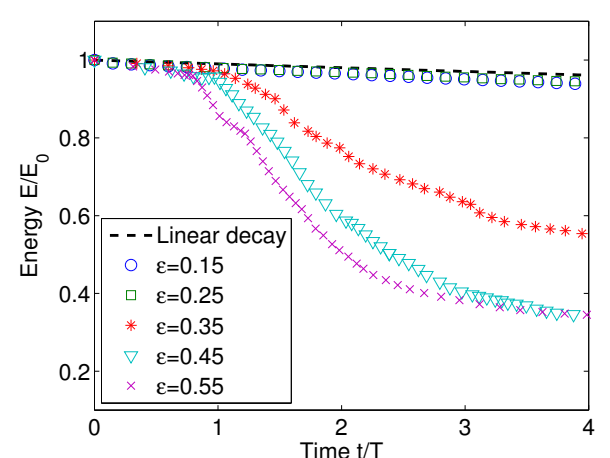

(b) $B o=1000$

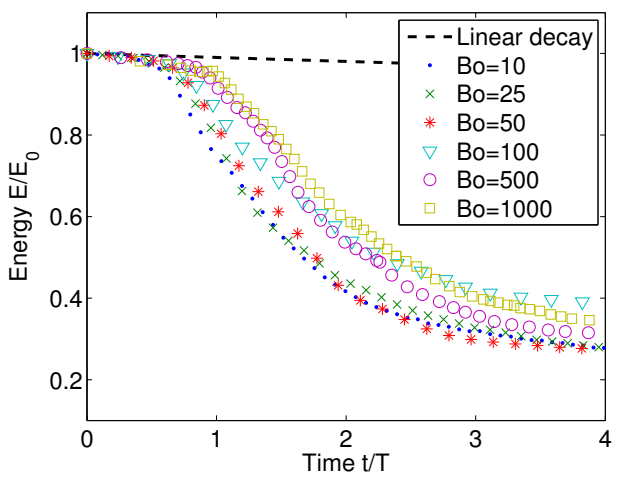

(d) $\epsilon=0.45$

Figure 9: Normalized wave energy $E / E_{0}$ as a function of time $t / T$. Figure (a,b): effect of increasing steepness for (a) $B o=10$ and (b) $B o=1000$. From top to bottom $\epsilon=$ $0.15,0.25,0.35,0.45$ and 0.55 . Figure $(\mathrm{c}, \mathrm{d})$ : effect of increasing surface tension at a given steepness, (c) $\epsilon=0.3$ and $(\mathrm{d}) \epsilon=0.45$. From bottom to top $B o=2,5,10,20$ and 100 . Black dashed line is the theoretical linear viscous dissipation $E / E_{0}=\exp \left(-4 \nu k^{2} t\right)$.

of energy is enhanced, due to the appearance of parasitic capillaries. An increase of the surface tension energy is observed at this moment. The kinetic and potential energies oscillate with opposite phase at half the wave frequency, as previously observed. For the same value of $\epsilon=0.3$, when the surface tension is further increased $(B o=10$, see figure $8 \mathrm{e}$ ), the previous observations hold but with increased capillary effects. The surface tension energy during the appearance of the capillaries is higher and the dissipation rate is increased. The peak value of surface tension energy represents between 5 and $10 \%$ of the total dissipated energy of wave. These two cases correspond to gravity-capillary waves. For higher initial steepness $(\epsilon=0.45)$, a dramatic decrease of the energy is observed, corresponding to a spilling breaker (see figure 8d,f) and surface tension energy is up to $10 \%$ of the dissipated energy. Again, after the breaking, mostly kinetic energy remains in the system, while potential energy has been dissipated. For non-breaking waves, more energy is dissipated when the Bond number is lower, while for breaking waves, the total energy dissipated depends less on the Bond number. 


\subsection{Influence of Bo and $\epsilon$}

Figure 9 shows the decay of the total energy, $E / E_{0}$. Figures $9 \mathrm{a}$,b show the influence of the initial steepness at a given Bond number $(B o=10$ and $B o=1000$ respectively), with $\epsilon$ increasing from top to bottom. In the high Bond number case (almost no surface tension, $B o=1000)$, energy dissipation is increased for steepness higher than the breaking threshold $\left(\epsilon_{c}=0.32\right)$. For $\epsilon<\epsilon_{c}$, at high Bond number, the wave dissipation is close to the classical viscous dissipation. For $\epsilon>\epsilon_{c}$, a dramatic loss of energy is observed between $t / T=1$ and $t / T=2$ corresponding to the breaking event. The total energy dissipated after four wave periods increases with the initial steepness, for $\epsilon=0.35$ around $40 \%$ of the wave energy is lost while up to $70 \%$ is lost for $\epsilon=0.55$. In the case of strong surface tension effects $(B o=10)$, only the lowest steepness $(\epsilon=0.15)$ presents a decay close to the classical viscous decay. When the steepness is increased to $\epsilon=0.25$, energy dissipation is enhanced by the appearance of parasitic capillaries. The total dissipation is then further increased when the initial steepness is increased.

Breaking waves are thus characterized by a strong loss of energy during the breaking stage and the influence of the steepness is similar for low and high values of surface tension, the main difference being that in the presence of surface tension the critical steepness for which dissipation is enhanced is smaller, due to the appearance of parasitic capillaries.

Figures $9 \mathrm{c}, \mathrm{d}$ show the decay of the total energy $E / E_{0}$ and the influence of the surface tension at a given initial steepness ( $\epsilon=0.3$ and $\epsilon=0.45$ respectively).

At low steepness $(\epsilon<0.3)$ and without surface tension effects $(B o=1000)$, the wave dissipation is close to the classical theoretical dissipation (see figure 9) of a linear sinusoidal wave at small amplitude. When the Bond number decreases (surface tension increases), an abrupt decrease of the energy is observed around $t / T \approx 1$ (figure $9 \mathrm{c}$, $B o=10,25,50,100)$, which corresponds to the time when capillaries appear on the main gravity wave (see figure 3), while when surface tension is too low to induce the appearance of capillaries, the energy decay remains smooth (two top curve on figure $9 \mathrm{c}$, $B o=500,1000)$.

The appearance of parasitic capillaries induces a global enhancement of the wave energy dissipation. Moreover the final state of gravity-capillary waves depends on the intensity of the capillary effects: at $t / T=4,60 \%$ of the energy has been dissipated for $B o=10$, while $30 \%$ has been dissipated for $B o=25, \approx 20 \%$ for $B o=50,10 \%$ for $B o=100$, and only less than $5 \%$ can be attributed to the classical viscous dissipation. Thus, at steepness below the breaking threshold, capillary effects strongly increase the wave energy dissipation.

At higher steepnesses, $\epsilon=0.45$, (see figure $4 \mathrm{~d}$ ), wave breaking occurs and the total amounts of dissipated energy are similar for the various Bond numbers. Thus, even if the breaking process is qualitatively different (plunging or spilling wave), the total amount of energy dissipated is similar at high wave steepness.

Surface wave dissipation is thus first determined by the stability of the wave, given by the initial steepness. Breaking waves exhibit strong dissipation in agreement with previous results, both experimental and numerical. However, at smaller steepnesses, dissipation processes are strongly affected by the appearance of capillaries on the main propagating wave. Spilling breakers with high surface tension exhibit global dissipation of the same order as plunging breakers, while gravity-capillary waves show a global dissipation much higher than the one due to linear viscous dissipation, in agreement with Mui \& Dommermuth (1995); Fedorov \& Melville (1998). 


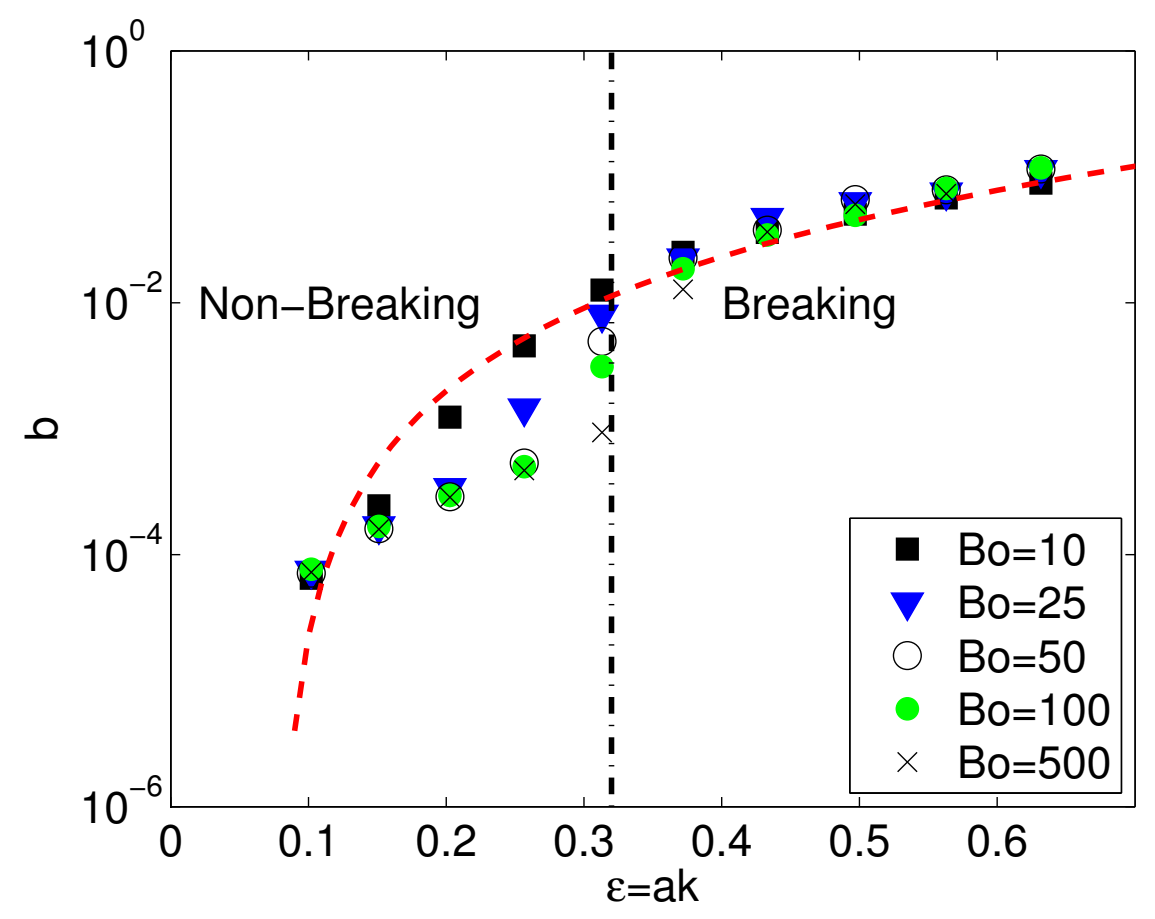

Figure 10: Breaking parameter $b$ as a function of steepness for various Bond numbers. Red dashed line is the semi-empirical dissipation function: $b=0.4(\epsilon-0.08)^{5 / 2}$ (eq. 4.6) from Romero et al. (2012). Vertical dot-dash line indicates the breaking threshold in the present work, $\epsilon_{c}=0.32$.

\subsection{Estimation of the breaking parameter $b$.}

We observe a dramatic decay of the wave energy during breaking events and the formation of parasitic capillaries, and showed that during these events, the decay has an exponential form $E \sim \exp [-\zeta(\epsilon, B o) t]$. We will now compare the decay rate obtained in the present simulations to experimental and theoretical results. The variation of the Bond number corresponds for air-water surface waves to various wavelengths, however, we have to keep in mind that the Reynolds number is constant in our study.

The dissipation induced by a breaking wave is usually described by the dissipation rate per unit length of breaking crest $\varepsilon_{l}$. Following Duncan (1981) and Phillips (1985),

$$
\varepsilon_{l}=b \rho_{w} c^{5} / g
$$

where $c$ is the phase velocity at breaking and $b$ is a non-dimensional parameter, called the breaking parameter. This formulation is the starting point for statistical modelling of wave breaking in the ocean (Phillips 1985; Romero et al. 2012). While the breaking parameter was first assumed to be constant (Phillips 1985), a large variation of the measured values of $b$ has been observed in various experiments. A scaling law, based on inertial arguments was derived for plunging waves (Drazen et al. 2008) and leads to the following result $b \sim S^{-5 / 2}$, where $S$ is the slope of the breaking wave. This result was then extended to spilling breakers by Pizzo \& Melville (2013). By assuming a threshold for breaking, the following general parametrization was supported by laboratory mea- 
surements and used for ocean wave modeling (Romero et al. 2012; Grare et al. 2013):

$$
b=0.4(S-0.08)^{5 / 2} .
$$

The dissipation rate per unit length $\varepsilon_{l}$ is simply given by $E_{0} \zeta$, where $E_{0}$ is the initial wave energy (with the dimension of an energy per unit length) and $\zeta$ the observed decay rate during breaking. The velocity $c$ is estimated by the linear phase velocity of the gravity-capillary wave $c=\sqrt{\frac{g}{k}+\frac{\gamma}{\rho_{w}} k}$. Thus $b=\varepsilon_{l} g /\left(\rho_{w} c^{5}\right)=E_{0} \zeta g /\left(\rho_{w} c^{5}\right)$, is shown in figure 10 as a function of the wave slope $\epsilon$ for all the breaking waves, the gravity-capillary waves and non-breaking waves. At steepness higher than the breaking threshold observed in these simulations $\epsilon>0.32$, a very good agreement is observed between the numerical results and the semi-empirical $\sim \epsilon^{5 / 2}$ scaling. Moreover the value of $b$ at high steepness appears independent of the Bond number, which is consistent with experimental results where $b$ is independent of the wavelength (Drazen et al. 2008; Romero et al. 2012; Grare et al. 2013). For non-breaking waves $(\epsilon<0.32)$ the definition and estimation of $b$ is not meaningfull which explains the observed discrepancy in figure 10. However it is remarkable to note that for the strong capillary effects cases, $B o=10$, and $B o=25$, the values of $b$ at low steepness are in good agreement with the semi-empirical curve for breaking. Thus, for these numerical solutions, the increase of dissipation induced by capillarity at low Bond number is consistent with the experimental scaling for breaking.

\section{Discussion on the wave regime diagram}

We will now discuss in more detail the application of the state diagram shown in figure 7 to waves observed in published experiments. Table 1 summarizes experimental observations of breaking and parasitic-capillary waves, for which both the slope and the Bond number could easily be extracted. The Bond number is estimated from the wavelength and the liquid properties $\left(B o=\rho g /\left(\gamma k^{2}\right)\right)$, and we also indicate the Reynolds numbers of the experiments $\left(R e=\sqrt{g \lambda^{3}} / \nu\right)$, which are often larger (especially for wave breaking) than the one used in the present simulations.

In these experiments, the waves are either generated by wind or by a wave maker. Breaking waves are either obtained through a focusing wave packet or are triggered by a modulational instability. In the first case, a wave-packet is generated by the wave maker, defining the central wavelength and the linear slope (through the sum of all frequency components of the initial packet). This slope is close (slightly smaller in general) to the slope at breaking that can be directly measured. This protocol has been used to study both spilling and plunging breakers. On the other hand, breaking triggered by the modulational instability corresponds to the instability of an initial wave (of a given wavelength) and the slope indicated in table 1 corresponds to the initial linear slope. In this case, the distinction between spilling and breaking wave is not necessarily done in the original papers and we will simply call these waves breakers. In the case of mechanically generated parasitic-capillary waves, both the wavelength and amplitude of the carrier wave are initial parameters of the experiment. Finally, for the wind generated waves, both the wavelength and the amplitude can be extracted from the figures of Zhang (1995) and Caulliez (2013).

Figure 11 shows the boundaries of the wave regime diagram of figure 7 , together with the experimental data summarized in table 1. For simplicity, we do not distinguish the various studies in the diagram but only the observed wave state, breaking triggered by modulation instability, plunging breakers, spilling breakers and parasitic capillary waves.

The first obvious observation is that, at high Bond number (i.e. large wavelength) 


\begin{tabular}{|c|c|c|c|c|c|c|c|}
\hline Reference & $\begin{array}{l}\text { Wave } \\
\text { type }\end{array}$ & $\lambda(\mathrm{m})$ & Bo & Slope (ak) & $\operatorname{Re}$ & Generation & Measurement \\
\hline $\begin{array}{l}\text { Rapp \& } \quad \& \\
\text { Melville } \\
(1990)\end{array}$ & Plunger & 1 to 2 & $\begin{array}{l}3000 \text { to } \\
12000\end{array}$ & 0.35 to 0.55 & $\begin{array}{l}3 \text { to } 9 \\
\times 10^{6}\end{array}$ & $\begin{array}{l}\text { Wave } \\
\text { maker }\end{array}$ & $\begin{array}{l}\text { Camera \& } \\
\text { wave gauges }\end{array}$ \\
\hline- & Spiller & 1 to 2 & - & 0.25 to 0.32 & - & - & - \\
\hline $\begin{array}{l}\text { Drazen et al. } \\
(2008)\end{array}$ & Plunger & 1 to 2 & $\begin{array}{l}3000 \text { to } \\
12000\end{array}$ & 0.35 to 0.55 & $\begin{array}{l}3 \text { to } 9 \\
\times 10^{6}\end{array}$ & $\begin{array}{l}\text { Wave } \\
\text { maker }\end{array}$ & $\begin{array}{l}\text { Camera \& } \\
\text { wave gauges }\end{array}$ \\
\hline $\begin{array}{l}\text { Duncan } \\
\text { et al. }(1999)\end{array}$ & Spiller & 0.77 & 2105 & 0.31 & $2 \times 10^{6}$ & $\begin{array}{l}\text { Wave } \\
\text { maker }\end{array}$ & Camera \\
\hline- & Spiller & 1.18 & 5000 & 0.31 & $4 \times 10^{6}$ & - & - \\
\hline $\begin{array}{l}\text { Duncan } \\
(2001)\end{array}$ & Spiller & 0.41 & 600 & 0.275 & $8 \times 10^{5}$ & $\begin{array}{l}\text { Wave } \\
\text { maker }\end{array}$ & Camera \\
\hline $\begin{array}{l}\text { Liu \& Dun- } \\
\text { can } \\
2006)\end{array}$ & Spiller & $\begin{array}{ll}0.4 & \text { to } \\
0.65 & \end{array}$ & $\begin{array}{l}1000 \\
1750\end{array}$ & 0.317 & $\begin{array}{l}8 \text { to } 16 \\
\times 10^{5}\end{array}$ & $\begin{array}{l}\text { Wave } \\
\text { maker }\end{array}$ & Camera \\
\hline $\begin{array}{l}\text { Fedorov } \\
\text { et al. }(1998)\end{array}$ & P-C-W & 0.1 & 35 & $0.28,0.29,0.33$ & $10 \times 10^{4}$ & $\begin{array}{l}\text { Wave } \\
\text { maker }\end{array}$ & $\begin{array}{l}\text { Wave } \\
\text { gauges }\end{array}$ \\
\hline- & P-C-W & 0.07 & 15 & $0.2,0.22,0.25$ & $5.8 \times 10^{4}$ & - & - \\
\hline- & P-C-W & 0.05 & 8 & $0.11,0.16,0.2$ & $3.5 \times 10^{4}$ & - & - \\
\hline $\begin{array}{l}\text { Caulliez } \\
(2013)\end{array}$ & Spiller & $\begin{array}{l}0.12 \\
0.17\end{array}$ & 50,100 & $0.34,0.37$ & $\begin{array}{l}13,22 \times \\
10^{4}\end{array}$ & Wind & Camera \\
\hline- & P-C-W & $\begin{array}{l}0.05 \text { to } \\
0.12\end{array}$ & 9 to 50 & 0.26 to 0.42 & $\begin{array}{l}3.5,13 \times \\
10^{4}\end{array}$ & - & - \\
\hline- & plunger & 0.25 & 215 & 0.5 & $39 \times 10^{4}$ & - & - \\
\hline $\begin{array}{l}\text { Zhang } \\
(1995)\end{array}$ & P-C-W & $\begin{array}{l}0.075 \\
0.05\end{array}$ & 19,9 & $0.21,0.25$ & $\begin{array}{l}3.5,6 \times \\
10^{4}\end{array}$ & Wind & Camera \\
\hline $\begin{array}{l}\text { Perlin et al. } \\
(1993)\end{array}$ & P-C-W & $\begin{array}{l}0.068 \\
0.105\end{array}$ & 16,38 & $0.22,0.24$ & $\begin{array}{l}3.5,10 \times \\
10^{4}\end{array}$ & $\begin{array}{l}\text { Wave } \\
\text { maker }\end{array}$ & Camera \\
\hline $\begin{array}{l}\text { Jiang et al. } \\
\text { (1999) }\end{array}$ & P-C-W & 0.101 & 35 & 0.11 to 0.25 & $10 \times 10^{4}$ & $\begin{array}{l}\text { Wave } \\
\text { maker }\end{array}$ & Camera \\
\hline- & P-C-W & 0.073 & 18 & 0.08 to 0.22 & $6 \times 10^{4}$ & - & - \\
\hline- & P-C-W & 0.066 & 15 & 0.1 to 0.21 & $5 \times 10^{4}$ & - & - \\
\hline- & P-C-W & 0.05 & 8.6 & 0.03 to 0.15 & $3.5 \times 10^{4}$ & - & - \\
\hline- & P-C-W & 0.045 & 7 & 0.13 to 0.16 & $3 \times 10^{4}$ & - & - \\
\hline $\begin{array}{l}\text { Banner \& } \\
\text { Peirson } \\
(2007)\end{array}$ & $\begin{array}{l}\text { Breakers } \\
(\mathrm{MI})\end{array}$ & $\begin{array}{l}0.38 \text { to } \\
0.46 \mathrm{~m}\end{array}$ & $\begin{array}{l}500 \\
766\end{array}$ & 0.11 to 0.17 & $\begin{array}{l}30^{6} \\
\text { to } 9\end{array}$ & $\begin{array}{l}\text { Wave } \\
\text { maker }\end{array}$ & $\begin{array}{l}\text { Wave } \\
\text { gauges }\end{array}$ \\
\hline $\begin{array}{l}\text { Tulin } \quad \& \\
\text { Waseda } \\
(1999)\end{array}$ & $\begin{array}{l}\text { Breakers } \\
(\mathrm{MI})\end{array}$ & 0.4 to 4 & $\begin{array}{l}500 \text { to } \\
50000\end{array}$ & 0.1 to 0.3 & $\begin{array}{l}0.8 \text { to } 20 \\
\times 10^{6}\end{array}$ & $\begin{array}{l}\text { Wave } \\
\text { maker }\end{array}$ & $\begin{array}{l}\text { Wave } \\
\text { gauges }\end{array}$ \\
\hline
\end{tabular}

Table 1: Experimental parameters of observed parasitic capillary waves, spilling and plunging breakers observed in the literature. The generation and measurement techniques are briefly summarized. Breaking waves due to the modulation instability are indicated (MI) and the distinction between spilling or plunging breakers is not made in these papers. 


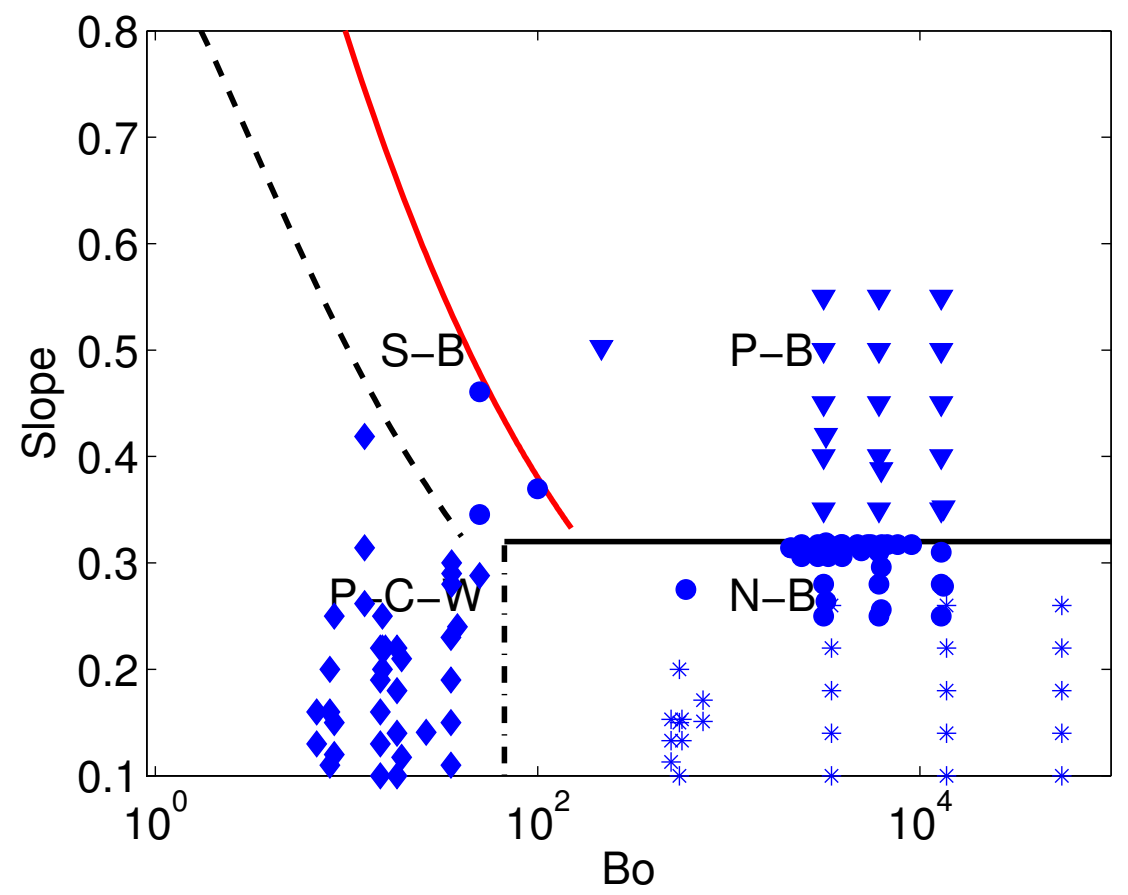

Figure 11: Wave state diagram, compares the regimes obtained through the simulation and described in fig. 7 with the experimental studies summarized in table 1. Experimental studies: $*$ : breakers triggered by modulation instability, $(\boldsymbol{\nabla})$ : plunging breakers, $(\bullet)$ : spilling breakers, $(\checkmark)$ : parasitic capillary waves

breaking is observed in numerous experiments for much smaller initial values of the wave slope than what is described in the present numerical work. Breaking through modulational instabilies is observed from $\epsilon \sim 0.11$ and spilling breakers are observed in focusing experiments for $\epsilon \sim 0.25$. Our breaking threshold at $\epsilon_{c}=0.32$ is thus related to our initial conditions. However, this allows observation of a critical value of the Bond number necessary for the influence of surface tension in the wave dynamics. The plunging breakers generated in focusing experiments have very high steepness $(\epsilon>0.35)$ and high Bond numbers $(B o>1000)$ and are indeed located in the plunging regime of our diagram (P-B).

Note that most of the spilling breakers described experimentally (Rapp \& Melville 1990; Duncan 2001; Liu \& Duncan 2003, 2006) correspond to relatively high Bond number $(B o>1000)$ and have typical slopes $\epsilon \in[0.25: 0.35]$ close to our critical steepness. This tends to confirm the idea that spilling breakers at large Bond number (large scale) is a transition regime between non-breaking and plunging wave. On the other hand, the wind generated small scale spilling breakers described by Caulliez (2013) correspond to smaller Bond number $(B o<100)$ and larger slope and are located inside the spilling zone (S-B).

Finally, at low Bond number, the experimentally observed parasitic-capillary waves have Bond numbers smaller than $B o_{c}$ and a slope smaller than the breaking steepness, $\epsilon<\epsilon_{B}=K_{B}(1+B o)^{-1 / 3}$. However, only a few data points exist at high steepness close to the boundary $\epsilon_{B}$. 
To conclude this comparison, when surface tension effects are important $(B o<100)$, the phase diagram described by the numerical simulation appears compatible with numerous experimental observations of parasitic capillary waves and small-scale spilling breakers. However, further studies are needed to validate experimentally the scaling of the breaking and the spilling-plunging boundaries at high steepness, $\epsilon \sim(1+B o)^{-1 / 3}$.

The present DNS have been performed assuming a uniform surface tension. The presence of surfactants on the air-water interface can modify the wave dynamics. Liu \& Duncan $(2003,2006)$ have investigated the influence of surfactants experimentally and found that adding surfactants significantly changes the breaking process. For high surfactant concentration, they observe a small plunging jet issued from the front face of the wave at a point below the wave crest. Note that the data from Liu \& Duncan $(2003,2006)$ are included in Fig. 11 and their spilling breaker for various surfactant concentration are inside the spilling phase. Liu \& Duncan $(2003,2006)$ also observed that the capillary ripples found upstream of the spilling breaker toe in the clean-water case are dramatically reduced for all concentrations of surfactants. Similar results were found by Ceniceros (2003) who investigated numerically the effect of surfactant on parasitic capillaries.

\section{Conclusion}

A parametric study of the influence of the wave steepness and Bond number on the surface wave evolution is presented, using direct numerical simulations of the two-phase flow, in air and water, including surface tension and viscosity. The study is conducted at a given high Reynolds number in order to study the effects of surface tension on wave breaking. A regime diagram is presented in which we report the observation of pure gravity waves, gravity-capillary waves, plunging breakers and spilling breakers. We are able to go continuously from one wave pattern to another by changing the Bond number and the initial steepness of the wave. For our initial conditions, we observe that when surface tension effects are small, a critical steepness $\epsilon_{c}$ can be defined to distinguish breaking from non-breaking waves, as already discussed theoretically as well as through experiments and numerical studies (Rapp \& Melville 1990; Peregrine et al. 1993; Song \& Banner 2002; Banner \& Peirson 2007; Perlin et al. 2013). When surface tension becomes important, its influence is quantified through two boundaries separating firstly gravity-capillary waves and breakers, and secontly spilling and plunging breakers. Both boundaries scale as $\epsilon \sim(1+B o)^{-1 / 3}$. This scaling is consistent with a balance between the generation of vorticity by gravity-capillary waves (Longuet-Higgins 1992) and the pressure due to capillary effects. When surface tension effects are important, our regime diagram appears compatible with the available experimental data.

The wave energy is computed for each wave state and confirms the importance of breaking processes in surface wave dissipation. Plunging and spilling breakers both exhibit dramatic loss of energy during the breaking stage, with little potential energy remaining in the system. This is a result of the periodic boundary conditions, in an open system we would expect equipartition in the surviving wave field. At low steepness, the wave dissipation is strongly enhanced when surface tension effects are important, while decay rates are comparable in the case of breaking waves for various Bond numbers. Moreover, from the wave energy decay rate during breaking, we are able to estimate the breaking parameter $b$ and our simulations appear fully consistent with the inertial scaling for breaking waves $b \sim \epsilon^{5 / 2}$ obtained using scaling arguments and observed experimentally (Drazen et al. 2008; Romero et al. 2012; Grare et al. 2013). The fact that non-breaking parasitic capillaries exhibit dissipation rates compatible with the breaking dissipation scaling is a very promising result that deserves further investigations. Note 
that these results are in qualitative agreement with the recent study of dissipation due to parasitic capillaries by Melville \& Fedorov (2014) based on the theory of Fedorov \& Melville (1998).

In this study, we have focused on the influence of surface tension, by changing the Bond number and keeping the Reynolds number constant. A natural development would be to now to study waves of various wavelengths, which would allow a closer comparison with experimental results. Furthemore, this has been a two-dimensional study and three dimensional simulations are required to confirm the evolution of the resulting turbulent flows.

We thank Murray Smith and Nick Pizzo for helpful discussions. This work was started by LD when a visiting student at NIWA and completed as a postdoctoral researcher at SIO. At SIO, LD and WKM were supported by grants to WKM from NSF (OCE) and ONR (Physical Oceanography).

\section{REFERENCES}

Agbaglah, G., Delaux, S., Fuster, D., Hoepffner, J., Josserand, C., Popinet, S., Ray, P., Scardovelli, R. \& Zaleski, S. 2011 Parallel simulation of multiphase flows using octree adaptivity and the volume-of-fluid method. Comptes Rendus Mecanique 339 (23), $194-207$.

Bague, A., Fuster, D., Popinet, S., Scardovelli, R. \& Zaleski, S. 2010 Instability growth rate of two-phase mixing layers from a linear eigenvalue problem and an initial value problem. Physics of Fluids 22 (9), 092104.

Banner, M.L. \& Peirson, W. L. 2007 Wave breaking onset and strength for two-dimensional deep-water wave groups. J. Fluid Mech. 585 (1), 93-115.

Batchelor, G. K. 1967 An introduction to fluid dynamics. Cambridge University Press.

Blenkinsopp, CE \& Chaplin, JR 2007 Void fraction measurements in breaking waves. Proc. R. Soc. A 463 (2088), 3151-3170.

Caulliez, G. 2013 Dissipation regimes for short wind waves. J. Geophys. Res. Oceans 118 (2), $672-684$.

Caulliez, G., Ricci, N. \& Dupont, R. 1998 The generation of the first visible wind waves. Physics of fluids $\mathbf{1 0 .}$

Ceniceros, H. D. 2003 The effects of surfactants on the formation and evolution of capillary waves. Physics of Fluids (1994-present) 15 (1), 245-256.

Chen, G., Kharif, C., Zaleski, S. \& Li, J. 1999 Two dimensionnal navier stokes simulation of breaking waves. Physics of fluid 11, 121-133.

CRAPPER, G. D. 1957 An exact solution for progressive capillary waves of arbitrary amplitude. J. Fluid Mech. 2, 532-540.

DiAs, F. \& Kharif, C. 1999 Nonlinear gravity and capillary-gravity waves. Annu. Rev. Fluid. Mech. 31, 301-346.

Douady, S., Couder, Y. \& Brachet, M. E. 1991 Direct observation of the intermittency of intense vorticity filaments in turbulence. Phys. Rev. Lett. 67, 983-986.

Drazen, D. A., Melville, W. K. \& Lenain, L. 2008 Inertial scaling of dissipation in unsteady breaking waves. J. Fluid Mech. 611 (1), 307-332.

DunCAN, JH 1981 An experimental investigation of breaking waves produced by a towed hydrofoil. Proceedings of the Royal Society of London. A. Mathematical and Physical Sciences 377 (1770), 331-348.

Duncan, J. H. 2001 Spilling breakers. Annu. Rev. Fluid. Mech. 33, 519-547.

Duncan, J. H., Qiao, H. \& Philomin, V. 1999 Gentle spilling breakers : crest profile evolution. J.Fluid Mech 379, 191-222.

Fedorov, A. V. \& Melville, W. K. 1998 Non linear gravity-capillary waves with forcing dissipation. J. Fluid Mech. 354, 1-42.

Fedorov, A. V., Melville, W. K. \& Rozenberg, A. 1998 An experimental and numerical study of parasitic capillary waves. Physics of Fluids 10 (6). 
Fenton, J. D. 1988 The numerical solution of steady water wave problems. Computers and Geosciences 14, 357-368.

Fructus, D., Clamond, D., Grue, J. \& Kristiansen, O. 2005 An efficient model for threedimensionnal surface wave simulations. part 1 : Free space problems. Journal of Computational Physics 205, 685-705.

Furhman, D. R., Madsen, P. A. \& Bingham, H. B. 2004 A numerical study of crescent wave. J.Fluid Mech 513, 309-341.

Fuster, D., Agbaglah, G., Josserand, C., Popinet, S. \& Zaleski, S. 2009 Numerical simulation of droplets, bubbles and waves: state of the art. Fluid Dyn Res 41, 065001.

Fuster, D., Matas, J.-P, Marty, S., Popinet, S., Hoepffner, J., Cartellier, A. \& ZALESKI, S. 2013 Instability regimes in the primary breakup region of planar coflowing sheets. J. Fluid Mech. 736, 150-176.

Grare, L., Peirson, W. L., Branger, H., Walker, J. W., Giovanangeli, J-P. \& Makin, V. 2013 Growth and dissipation of wind-forced, deep-water waves. J. Fluid Mech. 722, $5-50$.

IAfRATI, A. 2009 Numerical study of the effects of the breaking intensity on wave breaking flows. J. Fluid Mech. 622, 371-411.

IAFRATI, A 2011 Energy dissipation mechanisms in wave breaking processes: Spilling and highly aerated plunging breaking events. Journal of Geophysical Research: Oceans (1978-2012) $116(\mathrm{C} 7)$.

IAfrati, A, BABAnin, A \& Onorato, M 2013 Modulational instability, wave breaking, and formation of large-scale dipoles in the atmosphere. Phys. Rev. Lett. 110 (18), 184504.

IAfrati, A. \& CAmpanA, E.F. 2005 Free-surface fluctuations behind microbreakers: Spacetime behaviour and subsurface flow field. J. Fluid Mech. 529, 311-347.

JiAng, Lei, Lin, H-J., Schultz, W. W. \& Perlin, M. 1999 Unsteady ripple generation on steep gravity capillary waves. J. Fluid Mech. 386, 281-304.

Lamarre, E. \& Melville, W.K. 1991 Air entrainment and dissipation in breaking waves. Nature 351, 469-472.

Lamarre, Eric \& Melville, W. K. 1994 Voidfraction measurements and soundspeed fields in bubble plumes generated by breaking waves. J. Acoust. Soc. Am. 95 (3), 1317-1328.

Lamb, H. 1932 Hydrodynamics. Cambridge University Press.

Lin, J.C. \& Rockwell, D. 1995 Evolution of a quasi-steady breaking wave. J. Fluid Mech. 302, 29-44.

LiU, Xinan \& Duncan, J. H. 2003 The effects of surfactants on spilling breaking waves. Nature 421, 520-523.

Liu, Xinan \& Duncan, J. H. 2006 An experimental study of surfactant effects on spilling breakers. J. Fluid Mech. 567, 433-455.

Loewen, M. R., O’Dor, M. A. \& SkAfel, M. G. 1996 Bubbles entrained by mechanically generated breaking waves. J. Geophys. Res. 101 (C9), 20759-20769.

Longuet-Higgins, M. S. 1963 The generation of capillary waves by step gravity waves. $J$. Fluid Mech. 16, 138-159.

Longuet-Higgins, M. S. 1992 Capillary rollers and bores. J. Fluid Mech. 240, 659-679.

Longuet-Higgins, M. S. \& TAnAKA, M. 1997 On the crest instabilities of steep surface waves. J. Fluid Mech. 336, 51-68.

MCLEAn, J. 1982 Instabilities of finite amplitude water waves. J.Fluid Mech 114, 315-330.

Melville, W.K. \& Matusov, P. 2002 Distribution of breaking waves at the ocean surface. Nature 417, 58-63.

Melville, W. K. 1982 The instability and breaking of deep-water waves. J. Fluid Mech. 115, $165-185$.

Melville, W. K. 1996 The role of surface wave breaking in air-sea interaction. Annual Review of Fluid Mechanics 28, 279-321.

Melville, W. K. \& Fedorov, A. V. 2014 The equilibrium dynamics and statistics of gravitycapillary waves. J. Fluid Mech. subjudice.

Melville, W. K. \& RApp, D. J. 1985 Momentum flux in breaking waves. Nature 317.

Melville, W. K., Veron, F. \& White, C. J. 2002 The velocity field under breaking waves: coherent structure and turbulence. J. Fluid. Mech. 454. 
Mui, R. C. \& Dommermuth, D. G. 1995 Vortex generation by deep-water breaking waves. Journal of Fluids Engineering 117, 355-36.

Peregrine, D. H., Cokelet, E. D. \& McIver, P. 1993 Wave breaking in deep water. Annual Review of Fluid Mechanics 25, 373-397.

Perlin, M., Choi, W. \& Tian, Z. 2013 Breaking waves in deep and intermediate waters. Annual Review of Fluid Mechanics 45, 115-145.

Perlin, M., Lin, H-J. \& Ting, C-L. 1993 On parasitic capillary waves generated by steep gravity waves: an experimental investigation with spatial and temporal measurements. $J$. Fluid Mech. 255, 597-620.

Phillips, O.M. 1985 Spectral and statistical properties of the equilibrium range in windgenerated gravity waves. J. Fluid Mech. 156 (1), 505-31.

Pizzo, N.E. \& Melville, W. K. 2013 Vortex generation by deep-water breaking waves. J. Fluid Mech. 734, 198-218.

Popinet, S. 2003 Gerris: a tree-based adaptative solver for the incompressible euler equations in complex geometries. Journal of Computational Physics 190, 572-600.

Popinet, S. 2009 An accurate adaptative solver for surface-tension-driven interfacial flows. Journal of Computational Physics 228, 5838-5866.

QiaO, H. \& Duncan, J.H. 2001 Gentle spilling breakers: crest flow field evolution. J. Fluid Mech. 439, 57-85.

RAPp, R. J. \& MELVILle, W. K. 1990 Laboratory measurements of deep water breaking waves. Phil. Trans. R. Soc. London 331.

RojAs, G. \& Loewen, M.R. 2010 Void fraction measurements beneath plunging and spilling breaking waves. J. Geophys. Res. 118, 08001.

Romero, L., Melville, W. K. \& Kleiss, Jessica M. 2012 Spectral energy dissipation due to surface wave breaking. J. Phys. Oceanogr 42, 1421-1441.

Schwartz, L. 1966 Mathematics for the Physical Sciences. Hermann.

Song, C. \& Sirviente, A. 2004 A numerical study of breaking waves. Physics of fluid 16, $26-49$.

Song, J-B. \& BAnner, M. L. 2002 On determining the onset and strength of breaking for deep water waves. part i: Unforced irrotational wave groups. Journal of physical oceanography 32 (9), 2541-2558.

Su, M-Y. 1982 Three dimensionnal deep water waves. part 1. experimental measurement of skew and symetric wave patterns. J.Fluid Mech 124, 73-108.

Sutherland, P. \& Melville, W. K. 2013 Field measurements and scaling of ocean surface wave-breaking statistics. Geophysical Research Letters .

TsAi, W-T. \& Hung, L. 2007 Three-dimensionnal modeling of small-scale processes in the upper boundary layer bounded by a dynamic ocean surface. Journal of Geophysical Research 112, C02019.

Tsai, W-T. \& Hung, L. 2010 Enhanced energy dissipation by parasitic capillaries on short gravitycapillary waves. J. Phys. Oceanogr. 40, 2435-2450.

Tulin, M. P. \& WASEDA, T. 1999 Laboratory observations of wave group evolution, including breaking effects. J. Fluid Mech. 378, 197-232.

Veron, F., Melville, W.K. \& Lenain, L. 2008 Wave-coherent air-sea heat flux. J. Phys. Oceanogr 38, 788-802.

YAng, Y. \& Tryggvason, G. 1998 Dissipation of energy by finite amplitude surface waves. Computers and Fluids 27 (7), 829-845.

Zhang, S., Duncan, J.H. \& Chahine, G.L. 1993 The final stage of the collapse of a cavitation bubble near a rigid wall. J. Fluid Mech. 257, 147-181.

ZhANG, X. 1995 Capillarygravity and capillary waves generated in a wind wave tank: observations and theories. J. Fluid Mech. 289, 51-82. 